\title{
Modelling train-induced vibration of structures using a mixed-frame-of-reference approach
}

\author{
Paulius Bucinskas $^{1 *}$, Evangelos Ntotsios ${ }^{2}$, David J. Thompson ${ }^{2}$ and Lars V. Andersen ${ }^{1}$, \\ ${ }^{1}$ Department of Engineering, Aarhus University, Aarhus, 8000, Denmark \\ ${ }^{2}$ Institute of Sound and Vibration, University of Southampton, Southampton, SO17 1BJ, United Kingdom \\ *Corresponding author, e-mail address pb@eng.au.dk
}

\begin{abstract}
A novel computational modelling approach for prediction of environmental vibration is introduced. The model is formulated in both moving and fixed frames of reference, with a mixed frame of reference formulation introduced to couple the two frames of reference. The resulting system is able to model a vehicle travelling on an infinite railway track, formulated in a moving frame of reference, interacting via the soil with a structure (i.e. building), formulated in a fixed frame of reference. The method utilizes a semi-analytical soil model with the structures modelled using three-dimensional finite elements. Two solution procedures of the full system are proposed: partial coupling, where some secondary effects from reflected waves propagating through soil are disregarded, and full coupling, where the vehicle-track-soil-structure is modelled as a fully coupled system. Both proposed solution procedures offer a one-step approach for solving the whole system in the frequency-spatial domain. The usage of the model is demonstrated in two example cases: one analysing a simple building structure near a railway track, using the partial coupling solution procedure, and another one analysing the behaviour of a vehicle model traversing over a rigid block embedded inside the soil, using the full coupling solution procedure. The introduced modelling approach offers a computationally efficient solution procedure, at the same time being applicable to a wide array of application cases.
\end{abstract}

Keywords: soil-structure interaction, train induced vibration, mixed frame of reference

\section{Introduction}

Railway transportation has been seeing a resurgence in popularity in the recent years, as an efficient, fast and environmentally friendly form of transportation. This is especially evident in urban centres, where new metro and tramway lines are being continuously developed. However, constructing railway lines close to existing buildings or new buildings near existing lines leads to a set of challenges which need to be addressed. One of these challenges is the environmental vibration resulting from the passing trains. It propagates through the soil and enters the structures, causing annoyance to the inhabitants and, in worst case, leading to structural damage.

As shown in Connolly et al. [1], environmental vibration is a growing concern when planning new railway projects. The exact propagation mechanism of environmental vibration is an extremely complex phenomenon, and thus proper evaluation of its effects is difficult. A rough estimate of the vibration levels can be obtained by using the guidelines provided by, for example, the Federal Railroad Administration [2] that is commonly used in many countries. These estimates are generally based on empirical methods using on-site measurements. However, for more precise estimations, costly and time-consuming in-situ investigations need to be performed. Alternatively, computational models can also be used to evaluate these effects. Their use is advantageous as analysis can be performed even before the construction begins, and different configurations and site conditions can be tested. Nevertheless, detailed computational models often require sufficient engineering experience and long computation times, while simplified models assume significant reduction of site conditions, making their applications somewhat limited. 
When modelling environmental vibration, the modelling of the soil strata is, in most cases, the highest time consuming and difficult task. A sizable soil domain must be modelled to account for the source-soil-structure interaction, and in numerical models based on, for example, the finite element method (FEM), transmitting boundary conditions are required to ensure radiation of waves, thus mimicking the behaviour of an unbounded medium. This can be avoided to a degree by using analytical and semi-analytical approaches to model the underlying soil. Such models, utilizing purely analytical formulations of the Green's function, can be found in [3-5]. A widely used semi-analytical method, based on the soil layer transfer matrices formulated in frequency-wavenumber domain, was originally proposed by Thomson [6] and Haskell [7]. This semianalytical method offers relatively short computation times and ensures radiation without the need of special boundary conditions. The approach has been expanded by introducing rigid objects as described by Andersen and Clausen [8], or by coupling with a finite element (FE) model of external structures as described by Bucinskas and Andersen [9]. An analytical railway track model coupled to a semi-analytical soil model was introduced by Sheng et al. for a stationary load [10] and a moving load [11]. The railway track was modelled analytically as an infinite structure consisting of a beam and continuous springs and masses, this way accounting for the railway track behaviour at higher frequencies. The model was further expanded by introducing a multi-degree-of-freedom vehicle model and excitation from an uneven track [12]. The vehicle was modelled in two dimensions, accounting only for the vertical interactions between the wheels and the rails, with the whole system modelled in a moving frame of reference, and with the possibility to observe the resulting displacements at a non-moving receiver. The developed methodology offers relatively quick computations. However, the application range is limited by the assumptions made for the semi-analytical soil model (e.g. perfectly horizontal layers, homogeneity, isotropy etc.).

When more complex systems are investigated, the modelling effort increases accordingly. Three-dimensional (3D) problems can be analysed using the FEM. Examples of 3D FE models including surface railways can be found in [13-15]. The models can be formulated to account for a fully coupled vehicle-track-soil system, as shown by Connolly et al. [14], or a two-step approach can be used as shown by Kouroussis and Verlinden [15]. The biggest challenges of fully 3D models are the long computation times and the finite boundaries of the modelled domain. Another popular numerical approach is the boundary-element (BE) method. Like the semianalytical approach, this method has an inherent ability to radiate waves. Hence, only the boundaries of the investigated domain need to be discretized. The boundary-element method (BEM) is commonly coupled to the FEM, to create FE-BE models where the structures are modelled using the FEM and the soil is modelled using the BEM. Examples of 3D BE analysis and its coupling with the FEM can be found in [16-18]. Similar to fully 3D FE models, the 3D BE models still suffer from long computation times. This can be remedied to some degree by using so-called 'two-and-a-half-dimensional' (2.5D) models, originally proposed by Aubry et al. [19]. The methodology assumes invariant geometry along the movement direction of the vehicle, enabling Fourier transformation into wavenumber domain in this direction, in essence discretizing only the twodimensional (2D) cross section of the investigated domain. Then the 2D sections of the model are solved for different wavenumbers and, after inverse Fourier transformation, the 3D response can be obtained. The methodology is widely adopted and various approaches have been utilized to handle the non-reflecting boundary conditions: FE element models [20], FE-BE models [20, 21], FE with perfectly matched layers models (FE-PML) [23] or finite element-scaled-boundary-finite element models (FE-SBFEM) [24]. Only a small part of computational approaches and models developed over the years is presented here. A much wider overview of the methods used can be found in review papers, i.e. [25, 26] and in the comparative study of models by Connolly et al. [27].

When modelling the environmental vibration in a building induced by railway traffic, three main parts of the problem need to be considered: the vehicle-track system which is the vibration source, the underlying soil through which the vibration propagates, and the building structure which is the vibration receiver. Ideally, all the parts would be combined into a single model, including all coupling terms. However, due to complex analytical formulation needed and the limitations of computational models, this is rarely performed. Most 
often, parts of the model are coupled together; for example, a coupled vehicle-track-soil model is calculated, and the obtained results propagate to the building structure [22, 27-32]. This way, the secondary coupling terms are excluded from the model, such as the effect of the building structure to the response of the track and the vehicle. This is acceptable, as these coupling terms do not influence the system significantly in the majority of cases. For example, Fiala et al. [28] used a two-step approach to obtain the response of a building structure by splitting the problem into a vehicle-track-soil source model and a structure-soil receiver model. With a similar approach, François et al. [29] studied road-traffic-induced vibration. The work also proposed a methodology to exclude the soil-structure interaction problem for cases of soft structures resting on stiff soils. A so-called sub-modelling technique was proposed by Hussein et al. [31], where the response of the soil surface from a tunnel structure was modelled using the pipe-in-pipe method [34] to find the response of a 2D frame. An almost identical system assembly method was also used by Lopes et al. [23]; however, the soil surface response from a tunnel structure was modelled using a 2.5D FEM-PML model and a 3D building structure was analysed. Recently, Freisinger et al. [35] proposed a coupled Integral Transform Method (ITM) and FEM to model the soil structure interaction of finite spherical (3D) and length invariant cylindrical (2.5D) systems embedded or partly embedded in homogenous grounds.

Overall the prediction of environmental vibration using computational methods is a wide field, with a large variety of solutions developed over the years. However, an overall best method for the problem does not exist, and the decision on which approach to use depends largely on the available computational resources and acceptable level of simplification. The aim of this work is modelling the full vibration propagation path from the vehicle up to the building structure. The proposed novel method includes a moving vehicle model that is directly coupled to a stationary building structure, with interaction between them through the infinite underlying soil. At the same time, the method offers a relatively quick and flexible solution procedure. A fully 3D system is modelled, with structures modelled using FE, allowing a wide variety of configurations. To limit the computational times needed, the semi-analytical soil formulation is utilized, with a surface railway track, also modelled analytically as proposed by Sheng et al. [10-12]. The system is excited by a multi-body vehicle model passing over an irregular track. The work introduces an approach of solving the coupled vehicle-tracksoil-building system using a single step solution procedure. Two system assembly and solution methods are presented: partial coupling and full coupling. The partial coupling procedure disregards some effects caused by the reflected waves for a more computationally efficient solution. While, the full coupling procedure includes all the coupling terms present in the system. Additionally, the methodology for modelling rigid objects and FE structures interacting with the soil is described with its implementation into the proposed solution approaches.

The theoretical background of semi-analytical soil modelling is given in Section 2.1, while the relation between fixed and moving frame of reference (FOR) solutions is given in Section 2.2. The assembly of discretized soil and coupling it to FE is discussed in Section 3.1. Further, creating rigid objects interacting with the soil is introduced in Section 3.2. The vehicle-track system with the system excitation mechanism is introduced in Section 3.3. Section 4 presents the two assembly and solution procedures of a full system in a mixed FOR. The methodology is validated, by validating the analytically derived coupling terms, in Section 5.1, and comparing with another solution procedure, in Section 5.2. To show the capabilities of the proposed modelling approach, two example cases are analysed in Section 6. Finally, the conclusions can be found in Section 7.

\section{Semi-analytical soil model}

\subsection{Formulation in frequency-wavenumber domain}

A semi-analytical soil model is used in the present work. The model utilizes a well-known approach based on an analytical solution to the Green's function in the frequency-wavenumber domain. For the linear hysteric half-space $z \leq 0$, the displacement field in time-space domain can be obtained using a convolution integral 


$$
u_{i}(x, y, z, t)=\int_{-\infty}^{t} \int_{-\infty}^{0} \int_{-\infty}^{\infty} \int_{-\infty}^{\infty} g_{i j}\left(x-x^{\prime}, y-y^{\prime}, z, z^{\prime}, t-t^{\prime}\right) p_{j}\left(x^{\prime}, y^{\prime}, z^{\prime}, t^{\prime}\right) \mathrm{d} x^{\prime} \mathrm{d} y^{\prime} \mathrm{d} z^{\prime} \mathrm{d} t^{\prime}
$$

where the Green's function $g_{i j}$ relates the displacement component $u_{i}$ at the point $(x, y, z)$ and time $t$ to the loads $p_{j}$ applied in direction $j$ at all positions and times up to and including the time $t$. It should be noted that the Green's function is dependent explicitly on the vertical coordinates $z$ and $z^{\prime}$ of the observation and loading points and not only on the distance between the points in the depth direction. Thus, while the soil is assumed invariant and infinite in both horizontal directions, the material properties vary over depth due to stratification.

The Green's function is challenging to be calculated analytically in the time-space domain for a layered halfspace. Thus, a triple Fourier transformation is performed, transforming the two horizontal coordinates into the wavenumbers $k_{x}$ and $k_{y}$, and time into the circular frequency $\omega$. Further, introducing a discretization into a number of depths $z_{n}, n=1,2,3, \ldots, N_{z}$, Eq. (1) simplifies into

$$
\bar{U}_{i}\left(k_{x}, k_{y}, z, \omega\right)=\sum_{n=1}^{N_{z}} \bar{G}_{i j}\left(k_{x}, k_{y}, z, z_{n}, \omega\right) \bar{P}_{n, j}\left(k_{x}, k_{y}, \omega\right) .
$$

Here $\bar{U}_{i}$ and $\bar{G}_{i j}$ are components of the displacement vector and the Green's function tensor, respectively, in the frequency-wavenumber domain. After the discretization over depth, $\bar{P}_{n, j}$ signifies the traction applied on a horizontal interface placed at the depth $z_{n}$. In the following, upper case symbols indicate Fourier transforms with respect to time, whereas overbar indicates Fourier transforms with respect to the horizontal spatial coordinates.

Analytical expressions for the Green's function in frequency-wavenumber domain are available. Thomson [6] and Haskell [7] originally developed a transfer-matrix method, which relates the displacement and traction applied at the top of a single layer of material to the displacements and traction at the bottom of the same layer. For that purpose, it is assumed that every layer is composed of homogeneous and linear hysteretic material and that the layers are infinite in both horizontal directions. Further, interaction with the soil is only possible through the interfaces between layers or the soil surface. Using the transfer matrices, multiple layers of material are assembled and, after applying the boundary conditions, the expression for the Green's function can be obtained analytically. When the excitation loads are inside the homogenous layers, additional interfaces are included at each load depth. However, the assembly of multiple layers is not a straightforward task and requires further consideration in order to avoid numerical instabilities.

There are two major approaches used to assemble multiple soil layers: the flexibility approach and the stiffness approach. The flexibility approach is based on the original work proposed by Thomson [6] and Haskell [7]. The transfer matrices are multiplied together to propagate the displacements and tractions through the layers. Since the considered transfer matrices dimensions are never bigger than six by six (three displacement components and three traction components for three-dimensional wave propagation), the approach is very quick to compute, however it may suffer from instabilities due to ill-conditioned matrices occurring for problems where high frequencies or thick soil layers are considered. The stiffness approach was introduced by Kausel and Roesset [36] and extends the original transfer matrix, as derived by Thomson [6] and Haskell [7] by reordering it into a stiffness expression equivalent to those used in the FEM, assuming that the interface between two layers is interpreted as a connecting node. Multiple layers can be assembled by overlapping the stiffness matrices at the connecting interfaces. This approach leads to bigger matrices, especially when considering strata composed of many layers, however, the obtained system stiffness matrices are symmetric, and the approach does not suffer from the instability issues. The created system can then be used to obtain the Green's function of the system. 
The two methods described above perform very similarly and they can both be used to obtain the Green's function in the frequency-wavenumber domain providing identical results. In this work, both methods have been utilized: the flexibility approach with numerical stabilization for the fixed FOR soil model and the stiffness approach for the moving FOR model. The reason that the flexibility approach is used for the fixed FOR and the soil-structure interaction is because it enables the application of structure-to-soil interaction forces without increasing the size of the involved matrices (that is always six by six) for foundations that are embedded in the ground (i.e. piled foundations). For the moving FOR problem, only the Green's functions for a surface load (i.e. the track) are needed and thus the stiffness approach is used which is free of numerical instabilities at higher frequencies, that are more prominent due to the moving of the load.

The numerical instabilities and modelling errors that are possible to occur in the flexibility approach are caused respectively: (i) by the ill-conditioned matrices because the approach relates both traction and displacements components to each other in the same matrix and (ii) by the round-off error caused by exponentially increasing and decreasing terms in the same system because of the interaction between the primary (P) and the vertically polarized secondary (SV) waves propagating through the layers and reflecting at the interfaces. In the proposed model, the stabilization technique that is based on the orthonormalization method proposed by Wang [37] is used for coping with the any associated instabilities. Furthermore, very thick layers (if any) should be split into multiple smaller layers with the same material properties to not exceed the limits of floating-point numbers.

In the frequency-wavenumber domain, the displacements are calculated by multiplying the Green's function $\bar{G}_{i j}$ with the triple-Fourier-transformed load $\bar{P}_{n, j}$. Practise shows that using spatially distributed loads is advantageous, since such loads when transformed in wavenumber domain vanish at infinity. Contrarily, a concentrated force applied in a single spatial point produces a constant value of the Fourier transform for all wavenumbers-i.e. "white noise" in wavenumber domain - thus requiring, in principle, evaluation of the Green' function and inverse Fourier transformation up to infinite wavenumbers. A further discussion about the load distribution used in this work can be found in Section 5.2. After the displacements $\bar{U}_{i}$ in the frequencywavenumber domain are obtained, a double inverse Fourier transformation is performed into frequency-space domain:

$$
U_{\mathrm{f} i}\left(x_{\mathrm{f}}, y, z, \omega_{\mathrm{f}}\right)=\frac{1}{4 \pi^{2}} \int_{-\infty}^{\infty} \int_{-\infty}^{\infty} \bar{U}_{i}\left(k_{x}, k_{y}, z, \omega_{\mathrm{f}}\right) \mathrm{e}^{\mathrm{i}\left(k_{x} x_{\mathrm{f}}+k_{y} y\right)} \mathrm{d} k_{x} \mathrm{~d} k_{y} .
$$

The above equation is used when both the load and the response in a fixed FOR is considered. The load is applied at a circular frequency $\omega_{\mathrm{f}}$ and coordinate $x_{\mathrm{f}}$, with subscript ' $\mathrm{f}$ ' indicating a fixed FOR. As described by Andersen and Clausen [8], a load applied axisymmetrically around the point $\left(x^{\prime}, y^{\prime}, z^{\prime}\right)$ can be treated effectively in the fixed FOR. Here, the inverse Fourier transformation can be carried out in semi-discrete form by adopting polar coordinates, since the integration with respect to the azimuthal angle can be done in closed form, leading to Bessel functions in the components $G_{\mathrm{f} i j}$ of the Green's function.

When the whole system is considered in a moving FOR, the Green's function and, at the same time, the response in the frequency-wavenumber domain lose the polar symmetry around the origin of the wavenumber domain, compared to a purely fixed FOR. Hence, a fully discrete inverse Fourier transformation from wavenumber domain into spatial domain is necessary. Assuming that a load is moving in the positive $x$ direction, the frequency used to compute the Green's function becomes wavenumber dependent. In that case, the inverse double Fourier transformation into frequency-space domain is defined as

$$
U_{\mathrm{m} i}\left(x_{\mathrm{m}}, y, z, \omega_{\mathrm{m}}\right)=\frac{1}{4 \pi^{2}} \int_{-\infty}^{\infty} \int_{-\infty}^{\infty} \bar{U}_{i}\left(k_{x}, k_{y}, z, \omega_{\mathrm{m}}-k_{x} v\right) \mathrm{e}^{\mathrm{i}\left(k_{x} x_{\mathrm{m}}+k_{y} y\right)} \mathrm{d} k_{x} \mathrm{~d} k_{y}
$$

where $v$ is the velocity at which the moving FOR travels through the fixed FOR. Subscript ' $m$ ' indicates values in the moving FOR. 


\subsection{Relation between fixed and moving frames of reference}

The methodology presented in this work uses both moving and fixed frames of reference. Thus, a mixed FOR is used, where the displacement response observed in a fixed FOR from a load applied at a stationary point in the moving FOR and vice versa are needed in order to obtain the coupling terms between the two frames of reference.

Following a similar analysis with in [11], the displacements $U_{\mathrm{f} i}$ at $x_{\mathrm{f}}$ for fixed FOR from a harmonic moving load of circular frequency $\omega_{m}$ can be expressed as:

$$
U_{\mathrm{f} i}\left(x_{\mathrm{f}}, y, z, \omega_{\mathrm{m}}, \omega_{\mathrm{f}}\right)=\frac{1}{v} \mathrm{e}^{\mathrm{i} \beta x_{\mathrm{f}}} \frac{1}{2 \pi} \int_{-\infty}^{\infty} \bar{U}_{i}\left(\beta, k_{y}, z, \omega_{\mathrm{m}}-\beta v\right) \mathrm{e}^{\mathrm{i} k_{y} y} \mathrm{~d} k_{y}, \quad \beta=\frac{\omega_{\mathrm{m}}-\omega_{\mathrm{f}}}{v}
$$

where $\omega_{\mathrm{f}}$ is the receiving frequency at the fixed FOR.

Alternatively, using an equivalent analysis with [11], for a stationary harmonic load of circular frequency $\omega_{m}$ received in a moving FOR at $x_{\mathrm{m}}=x_{\mathrm{f}}-v t$ the displacements $U_{\mathrm{m} i}$ can be expressed as:

$$
U_{\mathrm{m} i}\left(x_{\mathrm{m}}, y, z, \omega_{\mathrm{f}}, \omega_{\mathrm{m}}\right)=\frac{1}{v} \mathrm{e}^{\mathrm{i} \beta x_{\mathrm{m}}} \frac{1}{2 \pi} \int_{-\infty}^{\infty} \bar{U}_{i}\left(\beta, k_{y}, z, \omega_{\mathrm{f}}\right) \mathrm{e}^{\mathrm{i} k_{y} y} \mathrm{~d} k_{y} .
$$

Comparing Eqs. (5) and (6) it is evident that the expressions are equivalent. That is, the displacements originated from a moving source and observed in a fixed FOR are equivalent to the displacements caused by a stationary source and observed in a moving frame:

$$
U_{\mathrm{m} i}\left(x_{\mathrm{m}}, y, z, \omega_{\mathrm{f}}, \omega_{\mathrm{m}}\right)=U_{\mathrm{f} i}\left(x_{\mathrm{f}}, y, z, \omega_{\mathrm{m}}, \omega_{\mathrm{f}}\right)
$$

given that the horizontal coordinates $x_{\mathrm{m}}$ and $x_{\mathrm{f}}$ have the same numerical value. In practise, this means that only Eq. (5) or Eq. (6) needs to be evaluated. Then the integral part of the equation is reused for the other FOR combination, with changed $x$-coordinate. It should be noted that this is only true in the mixed frequency domain. When inverse Fourier transformation is performed to obtain the time-domain response, this is no longer true, even if the circular frequencies $\omega_{\mathrm{m}}$ and $\omega_{\mathrm{f}}$ are equal:

$$
u_{\mathrm{m} i}\left(x_{\mathrm{m}}, y, z, \omega_{\mathrm{f}}, t\right) \neq u_{\mathrm{f} i}\left(x_{\mathrm{f}}, y, z, \omega_{\mathrm{m}}, t\right) .
$$

\section{Structures interacting with soil}

\subsection{Assembly in a single frame of reference}

To couple the semi-analytical soil model to a FE model of one or more structures, the dynamic stiffness matrix $\mathbf{K}_{\mathrm{ss}}(\omega)$ of the soil has to be established. The desired geometry of the soil-structure interface is discretized into a number of 'soil-structure interaction' (SSI) nodes and the response is requested at a range of 'observation' nodes. Thus, two types of nodes are used to model the system; the SSI nodes that are interacting with external structures, and the observation nodes which are only used to analyse soil displacements without direct interaction. The dynamic stiffness matrix is established using the SSI nodes, which is then coupled to structures and used to obtain the system displacements. The displacements of the observation nodes are obtained by postprocessing the previously obtained results.

The dynamic stiffness matrix $\mathbf{K}_{\mathrm{ss}}(\omega)$ of the soil is calculated by inverting the global receptance matrix $\mathbf{R}_{\mathrm{ss}}(\omega)$ relating all the degrees of freedom needed. For a three-dimensional case, each SSI node has three degrees of freedom. Therefore, the receptance matrix $\mathbf{R}_{\mathrm{ss}}(\omega)$, also called the flexibility matrix, will be a square matrix with number of rows and columns three times the number of nodes in the system. To create the receptance matrix, a unit harmonic load is applied to a single degree of freedom $s_{j}$ and the resulting displacements are calculated at all SSI degrees of freedom. The procedure is repeated for all SSI degrees of freedom in the system. The receptance between degrees of freedom $s_{i}$ and $s_{j}$ is given as 


$$
R_{S_{i}, S_{j}}(\omega)=\int_{-\infty}^{\infty} \int_{-\infty}^{\infty} \bar{G}_{d_{i} d_{j}}\left(k_{x}, k_{y}, z_{n_{i}}, z_{n_{j}}, \omega\right) \bar{P}_{n_{j}, S_{j}}\left(k_{x}, k_{y}, \omega\right) \mathrm{e}^{\mathrm{i}\left(k_{x}\left(x_{n_{i}}-x_{n_{j}}\right)+k_{y}\left(y_{n_{i}}-y_{n_{j}}\right)\right)} \mathrm{d} k_{x} \mathrm{~d} k_{y}
$$

where $\bar{P}_{n_{j}, s_{j}}\left(k_{x}, k_{y}, \omega\right)$ is the double spatial Fourier transform of a distributed harmonic load $P_{n_{j}, s_{j}}\left(x_{n_{j}}, y_{n_{j}}, z_{n_{j}}, \omega\right)$ of unit magnitude acting within the frequency domain at the depth $z_{n_{j}}$ in degree of freedom $s_{j}$. Corresponding to the SSI degrees of freedom $s_{i}$ and $s_{j}, d_{i}$ and $d_{j}$ signify the local degrees of freedom, $d_{i}, d_{j}=1$, 2, 3 for the three-dimensional solid, while $n_{i}$ and $n_{j}$ are the node numbers of the nodes to which SSI degrees of freedom $s_{i}$ and $s_{j}$ belong. Thus, it is assumed that the displacement is observed in direction $d_{i}$ at a node with coordinates $\left(x_{n_{i}}, y_{n_{i}}, z_{n_{i}}\right)$, while the load is applied in direction $d_{j}$ at a node with coordinates $\left(x_{n_{j}}, y_{n_{j}}, z_{n_{j}}\right)$. The same procedure can be performed in both fixed and moving frames of reference. The obtained results are placed in a single column of the receptance matrix and the process is repeated for every degree of freedom.

The receptance matrix $\mathbf{R}_{\mathrm{sS}}(\omega)$ is a $S \times S$ matrix providing the flexibility of the $S$ SSI degrees of freedom from loads applied to the same $S$ degrees of freedom. The receptance matrix can be established for the moving and fixed frames of reference. However, only in the fixed FOR, the matrix is symmetric, assuming that the applied load $P_{n_{j}, S_{j}}(x, y, \omega)$ is the same for all degrees of freedom.

The inversion of the flexibility matrix for large systems can be computationally expensive. Thus, using as few as possible SSI nodes is important for an efficient solution. To find the optimum number and placement of the nodes a convergence analysis is often required, especially when modelling rigid structures.

After the dynamic stiffness matrix is obtained, it can be assembled in the single frame of reference (SFOR) with the dynamic stiffness matrix $\mathbf{K}_{\mathrm{FE}}$ of the FE model:

$$
\mathbf{K}_{\mathrm{SFOR}}(\omega)=\left[\begin{array}{cc}
\mathbf{K}_{\mathrm{SS}}(\omega)+\mathbf{K}_{\mathrm{FE}}^{\mathrm{ss}}(\omega) & \mathbf{K}_{\mathrm{FE}}^{\mathrm{sn}}(\omega) \\
\mathbf{K}_{\mathrm{FE}}^{\mathrm{ns}}(\omega) & \mathbf{K}_{\mathrm{FE}}^{\mathrm{nn}}(\omega)
\end{array}\right],
$$

where superscript 's' denotes the degrees of freedom through which the FE model interacts with the soil, while superscript ' $n$ ' denotes the degrees of freedom that are internal to the FE model. Combinations of superscripts 'sn' and 'ns' denote the coupling terms. Due to symmetry of the FE system matrices, $\mathbf{K}_{\mathrm{FE}}^{\mathrm{ns}}(\omega)=\left[\mathbf{K}_{\mathrm{FE}}^{\mathrm{sn}}(\omega)\right]^{\mathrm{T}}$. Further, the matrix $\mathbf{K}_{\mathrm{FE}}^{\mathrm{sn}}(\omega)$ is usually sparsely populated.

The displacements of the system can then be obtained by solving the system of equations:

$$
\mathbf{K}_{\mathrm{SFOR}}(\omega) \mathbf{U}_{\mathrm{SFOR}}(\omega)=\mathbf{P}_{\mathrm{SFOR}}(\omega) .
$$

To obtain the displacements of the observation degrees of freedom, the flexibility matrix $\mathbf{R}_{\text {os }}$ relating the SSI and observation degrees of freedom is needed. Assuming that the numbering of observation degrees of freedom is stored in a set $o=\left\{o_{1}, o_{2}, \ldots, o_{O}\right\}$ with the number of degrees of freedom being $O$, the flexibility matrix will have $O$ rows and $S$ columns. The displacements of the observation nodes are given by multiplying the observation flexibility matrix $\mathbf{R}_{\mathrm{os}}$ with the soil displacements at the SSI degrees of freedom $\mathbf{U}_{\mathrm{ss}}$. These are extracted from the whole system displacement vector $\mathbf{U}_{\mathrm{SFOR}}$ :

$$
\mathbf{U}_{\mathrm{o}}(\omega)=\mathbf{R}_{\mathrm{os}}(\omega)\left[\mathbf{R}_{\mathrm{ss}}(\omega)\right]^{-1} \mathbf{U}_{\mathrm{s}}(\omega)=\mathbf{R}_{\mathrm{os}}(\omega) \mathbf{K}_{\mathrm{ss}}(\omega) \mathbf{U}_{\mathrm{ss}}(\omega)
$$

Since the matrix inversion involved in Eq. (12) has been already performed, the result can be reused. Thus, to obtain the displacements of the observation nodes no further large matrix inversion is needed, making the calculations more computationally efficient. Observation nodes are especially useful when the displacements of large fields are of interest, for example, to observe the displacement of the ground surface in a large region. 


\subsection{Rigid structures interacting with the soil}

The semi-analytical soil model allows modelling of completely rigid objects interacting with the soil. This is useful when modelling structures that are much stiffer than the surrounding material, as for example building foundations. To create a three-dimensional rigid object, the object shape is discretized into a number of SSI nodes. The global flexibility matrix is created in the same way as in the previous subsection and inverted to obtain the dynamic stiffness matrix. However, condensation of the stiffness matrix must be performed in order to reduce the system such that the response can be determined in terms of the rigid body modes rather than the original degrees of freedom of the SSI nodes. This is achieved by assuming that SSI nodes belonging to the same rigid object are fixed relatively to each other and move together with the degrees of freedom of a reference master node that defines the motion of the rigid object.

In principle, the master node can be placed at any position. However, it is most conveniently placed in the point at which coupling to an FE model should be done. That is, for example, in the centre of the topside of a footing. This way, the rotational degrees of freedom between the two systems can be coupled directly, without the need to derive additional rotational coupling terms. In the most common three-dimensional case, separate SSI nodes have three degrees of freedom each, i.e. three lateral displacements, while each rigid object has six degrees of freedom: three for lateral displacements and three additional rotational degrees of freedom of the reference node. For a single rigid object composed of a number of SSI nodes, the transformation matrix $\mathbf{T}_{0, i}$ is created. Multiple rigid objects can also be a part of the same system. The global transformation matrix for a system with $N$ rigid objects can be assembled as:

$$
\mathbf{T}_{\mathrm{S}}=\left[\begin{array}{cccc}
\mathbf{T}_{0,1} & \mathbf{0} & \cdots & \mathbf{0} \\
\mathbf{0} & \mathbf{T}_{0,2} & \cdots & \mathbf{0} \\
\vdots & \vdots & \ddots & \vdots \\
\mathbf{0} & \mathbf{0} & \cdots & \mathbf{T}_{0, N}
\end{array}\right] .
$$

Formulation for transformation matrix $\mathbf{T}_{0, i}$ can be found in previous works by various authors, e.g. [38]. The system can also contain non-associated 'free' SSI nodes, which are not part of any rigid object. In that case, the local transformation matrix for such nodes will be the identity matrix with the same number of rows and columns as the number of degrees of freedom associated with the free nodes. The condensed stiffness matrix of the soil is given by left and right matrix multiplication of $\left[\mathbf{R}_{\mathrm{ss}}(\omega)\right]^{-1}$ with $\left[\mathbf{T}_{\mathrm{s}}\right]^{\mathrm{T}}$ (where []$^{\mathrm{T}}$ is transpose matrix notation) and $\mathbf{T}_{\mathrm{s}}$ respectively. The resulting stiffness matrix can then be coupled to FE structures in the same way as described previously. Due to condensation of some SSI nodes, Eq. (12) is also modified by introducing the transformation matrix:

$$
\mathbf{U}_{\mathrm{o}}(\omega)=\mathbf{R}_{\mathrm{os}}(\omega)\left[\mathbf{R}_{\mathrm{ss}}(\omega)\right]^{-1} \mathbf{T}_{\mathrm{s}} \mathbf{U}_{\mathrm{s}}(\omega)=\mathbf{R}_{\mathrm{os}}(\omega) \mathbf{K}_{\mathrm{ss}}(\omega) \mathbf{T}_{\mathrm{s}} \mathbf{U}_{\mathrm{s}}(\omega) .
$$

Here the vector $\mathbf{U}_{\mathrm{s}}$ stores the already obtained displacements at the SSI degrees of freedom. The matrix inversion involved in Eq. (14) has been already performed and to improve the efficiency of the calculation, intermediate results should be saved and reused here. The formulation outlined here can be also used, if no rigid objects are present in the system. In that case each SSI node is treated as a 'free' node, creating a transformation matrix which is just a square identity matrix, with a number of rows and columns equal to the number of degrees of freedom in the system.

\subsection{Vehicle, railway track and wheel-rail interaction}

The system is excited by one or more vehicles travelling across a railway track. The vehicles can be modelled using various multibody systems, with varying complexity, depending in the application case. However, in the described model vehicles are only modelled in two dimensions and only the vertical wheel-rail interaction forces are considered. The dynamic stiffness matrix of the vehicle $\mathbf{K}_{\mathrm{vv}, i}\left(\omega_{\mathrm{m}}\right)$ is created in a moving FOR by combining the vehicle stiffness, damping and mass matrices. If multiple vehicles are needed, it is assumed that there is no direct interaction between vehicles and the dynamic stiffness matrix becomes: 


$$
\mathbf{K}_{\mathrm{v}}\left(\omega_{\mathrm{m}}\right)=\left[\begin{array}{cccc}
\mathbf{K}_{\mathrm{vv}, 1}\left(\omega_{\mathrm{m}}\right) & \mathbf{0} & \cdots & \mathbf{0} \\
\mathbf{0} & \mathbf{K}_{\mathrm{vv}, 2}\left(\omega_{\mathrm{m}}\right) & \cdots & \mathbf{0} \\
\vdots & \vdots & \ddots & \vdots \\
\mathbf{0} & \mathbf{0} & \cdots & \mathbf{K}_{\mathrm{vv}, I}\left(\omega_{\mathrm{m}}\right)
\end{array}\right],
$$

Here it is assumed that there is a total number $I$ of vehicles in the system. Further, there is no coupling between separate vehicles through the vehicle stiffness matrix, i.e. each car of a train acts like a separate vehicle.

A layered track structure containing the rails, rail-pads, sleepers and ballast is used. It is coupled to the underlying soil in the frequency-wavenumber domain as described in [39]. By using the stiffness matrix for the rails $\mathbf{K}_{\mathrm{r}}\left(\omega_{\mathrm{m}}\right)$ and the diagonal stiffness matrix $\mathbf{K}_{\mathrm{H}}$ for the wheel-rail contact interaction the coupled vehicle-track system response from a unit amplitude unevenness is given as:

$$
\left[\begin{array}{ccc}
\mathbf{K}_{\mathrm{v}}^{\mathrm{uu}}\left(\omega_{\mathrm{m}}\right) & \mathbf{K}_{\mathrm{v}}^{\mathrm{uw}}\left(\omega_{\mathrm{m}}\right) & \mathbf{0} \\
\mathbf{K}_{\mathrm{v}}^{\mathrm{wu}}\left(\omega_{\mathrm{m}}\right) & \mathbf{K}_{\mathrm{v}}^{\mathrm{ww}}\left(\omega_{\mathrm{m}}\right)+\mathbf{K}_{\mathrm{H}} & -\mathbf{K}_{\mathrm{H}} \\
\mathbf{0} & -\mathbf{K}_{\mathrm{H}} & \mathbf{K}_{\mathrm{r}}\left(\omega_{\mathrm{m}}\right)+\mathbf{K}_{\mathrm{H}}
\end{array}\right]\left\{\begin{array}{c}
\mathbf{U}_{\mathrm{v}}^{\mathrm{u}}\left(\omega_{\mathrm{m}}\right) \\
\mathbf{U}_{\mathrm{v}}^{\mathrm{w}}\left(\omega_{\mathrm{m}}\right) \\
\mathbf{U}_{\mathrm{r}}\left(\omega_{\mathrm{m}}\right)
\end{array}\right\}=\left\{\begin{array}{c}
\mathbf{0} \\
K_{\mathrm{H}} \mathbf{d}\left(\omega_{\mathrm{m}}\right) \\
-K_{\mathrm{H}} \mathbf{d}\left(\omega_{\mathrm{m}}\right)
\end{array}\right\} .
$$

where $\mathbf{d}\left(\omega_{\mathrm{m}}\right)=\exp \left(\frac{\omega_{\mathrm{m}}}{v} \mathbf{x}_{\mathrm{w}}\right)$ is the vector containing the unit unevenness for all wheel positions $\mathbf{x}_{\mathrm{W}}$ in the moving FOR. In Eq. (16), the vehicle degrees of freedom are split into two parts: those relating to the wheels, denoted with the superscript ' $w$ ', and those that are not coupled to the track, denoted with superscript ' $u$ '. It is assumed that each vehicle wheel has the same linearized Hertzian spring stiffness $K_{\mathrm{H}}$ when calculating the acting forces. Solving Eq. (16) for the unknown displacement vector $\mathbf{U}$ produces the system behaviour. Note that in this case, the effects of external structures coupled to the railway track through the soil are not accounted for.

The stiffness matrix for the rails in Eq. (16) can be found by inverting the flexibility matrix $\mathbf{R}_{\mathrm{rr}}\left(\omega_{\mathrm{m}}\right)$ relating the rail displacements between all vehicle wheel sets positions is constructed. This is achieved in a similar manner as described in the previous section, assuming a single degree of freedom of a rail in a moving FOR for every wheel. The matrix is later used to establish the stiffness matrix of a coupled-domain stiffness matrix.

\section{Assembly and solution of global system}

This section describes the assembly of the global system, coupling both moving and fixed FORs. The described assembly procedure can be used independent of the soil modelling method, as long as the receptance matrices used in the formulations found in Sections 3 and 4 can be obtained. However, it is advantageous to use the previously described semi-analytical formulation, as it produces the receptance matrices without the need of inverting large matrices. In the case of using a stiffness based approach, such as the FE or BE methods, a large matrix inversion is required to obtain the receptance matrices. In such cases, the analytically derived coupling terms could still be used to couple two FORs together.

\subsection{Partly coupled global system}

Parts of the model described in the previous sections are combined into a single global system. The resulting system combines parts formulated in a moving FOR, such as the vehicle and the track, and parts formulated in the fixed FOR, such as the structures interacting with the soil. To couple the two frames of reference together, the theory described in Sections 2.2 and 2.3 is used. For that purpose, the receptance matrix $\widetilde{\mathbf{R}}_{\text {sr }}$, providing the interaction between the degrees of freedom of the rails and the degrees of freedom of the soil, is established. The matrix $\widetilde{\mathbf{R}}_{\text {sr }}$ couples the moving and fixed frames of reference, and it is therefore dependent on two frequencies: $\omega_{\mathrm{m}}$ and $\omega_{\mathrm{s}}$. Accordingly, the tilde indicates that the quantity is defined in the mixed FOR.

The receptance defining the response of degree of freedom $i$ in the fixed FOR to a load applied to degree of freedom $j$ in the moving FOR can be found as: 


$$
\tilde{R}_{\mathrm{f} i, j}\left(\omega_{\mathrm{m}}, \omega_{\mathrm{f}}\right)=\frac{1}{v} \mathrm{e}^{\mathrm{i} \beta\left(x_{\mathrm{f} n_{i}}-x_{\mathrm{m} n_{j}}\right)} \frac{1}{2 \pi} \int_{-\infty}^{\infty} \bar{G}_{d_{i} d_{j}}\left(\beta, k_{y}, z_{n_{i}}, z_{n_{j}}, \omega_{\mathrm{m}}-\beta v\right) \bar{P}_{n_{j}, s_{j}} \mathrm{e}^{\mathrm{i}\left(y_{n_{i}}-y_{n_{j}}\right) k_{y}} \mathrm{~d} k_{y},
$$

where $d_{i}$ and $d_{j}$ are the directions associated with degrees of freedom $i$ and $j$, while $n_{i}$ and $n_{j}$ are the node numbers of the nodes to which degrees of freedom $i$ and $j$ belong. In this case, all loaded degrees of freedom belong to the rails and are applied as unit loads on an Euler-Bernoulli beam. Therefore, the load $\bar{P}_{n_{j}, S_{j}}$ is equal to one. The receptance matrix $\widetilde{\mathbf{R}}_{\mathrm{sr}}\left(\omega_{\mathrm{m}}, \omega_{\mathrm{f}}\right)$ can now be assembled and is a $S \times R$ matrix with $R$ the number of coupled rail degrees of freedom.Further, the receptance used for the backwards coupling from a load at degree of freedom $i$ in fixed FOR to a displacement in degree of freedom $j$ in a moving FOR is obtained. To avoid excessive additional computations, the similarity between the solutions for the two FOR combinations and the symmetry of the solution in the $y$-direction is used. Further, it is assumed that there is reciprocity of receptance between the rails and the soil. Thus, the coupling terms between loads applied in a stationary FOR and observed in a moving FOR can be found as:

$$
\tilde{R}_{\mathrm{m} i, j}\left(\omega_{\mathrm{f}}, \omega_{\mathrm{m}}\right)=\frac{1}{v} \mathrm{e}^{\mathrm{i} \beta\left(x_{\mathrm{m} n_{j}}-x_{\mathrm{f} n_{i}}\right)} \frac{1}{2 \pi} \int_{-\infty}^{\infty} \bar{G}_{d_{i} d_{j}}\left(\beta, k_{y}, z_{n_{i}}, z_{n_{j}}, \omega_{\mathrm{m}}-\beta v\right) \bar{P}_{n_{j}, S_{j}} \mathrm{e}^{\mathrm{i}\left(y_{n_{i}}-y_{n_{j}}\right) k_{y}} \mathrm{~d} k_{y} .
$$

By keeping the same integrand as in Eq. (17) and only modifying the $x$-coordinates, additional computations are avoided. The modification due to the $x$-coordinate is unavoidable as the solution is not symmetrical in the $x$-direction. The $S \times R$ receptance matrix $\widetilde{\mathbf{R}}_{\mathrm{rs}}\left(\omega_{\mathrm{f}}, \omega_{\mathrm{m}}\right)$ for backwards coupling can be assembled and then transposed, accounting for the switched source and receiver positions. However, as the effects of the backwards coupling in most cases are negligible, the transpose of the forwards coupling matrix, $\widetilde{\mathbf{R}}_{\text {sr }}\left(\omega_{\mathrm{m}}, \omega_{\mathrm{f}}\right)^{\mathrm{T}}$ can be also reused here with little loss of accuracy

Further, the flexibility matrix, assembled in the fixed FOR and connecting all the degrees of freedom interacting with the soil, is added to the system. Using the created matrices, the full flexibility matrix for the so-called 'global' (indicated by subscript 'g') system can be constructed:

$$
\widetilde{\mathbf{R}}_{\mathrm{g}}\left(\omega_{\mathrm{m}}, \omega_{\mathrm{f}}\right)=\left[\begin{array}{cc}
\mathbf{R}_{\mathrm{rr}}\left(\omega_{\mathrm{m}}\right) & \widetilde{\mathbf{R}}_{\mathrm{rs}}\left(\omega_{\mathrm{f}}, \omega_{\mathrm{m}}\right) \\
\widetilde{\mathbf{R}}_{\mathrm{sr}}\left(\omega_{\mathrm{m}}, \omega_{\mathrm{f}}\right) & \mathbf{R}_{\mathrm{ss}}\left(\omega_{\mathrm{f}}\right)
\end{array}\right] .
$$

When no rigid bodies are present in the soil model, the global flexibility matrix may be inverted to obtain the stiffness matrix $\widetilde{\mathbf{K}}_{\mathrm{g}}\left(\omega_{\mathrm{m}}, \omega_{\mathrm{f}}\right)$ of the global system.

If the system contains rigid objects, which are formulated as described in Section 3.2, the global stiffness matrix can be constructed using the global transformation matrix:

$$
\mathbf{T}_{\mathrm{g}}=\left[\begin{array}{ll}
\mathbf{I} & \mathbf{0} \\
\mathbf{0} & \mathbf{T}_{\mathrm{s}}
\end{array}\right]
$$

where I is the identity matrix with dimensions equal to the number of rail degrees of freedom. It is assumed that there are no rigid bodies modelled in the moving FOR. In theory, it is possible to model rigid bodies in both the moving and the fixed frames of reference, given that the reference nodes for these bodies are not shared between the two frames of reference. In that case, the identity matrix would be replaced with local transformation matrices $\mathbf{T}_{0, i}$. However, for any practical applications, rigid bodies will only exist in the fixed FOR, where they may be used to model foundations or structures embedded in the ground. The global transformation matrix $\widetilde{\mathbf{K}}_{\mathrm{g}}\left(\omega_{\mathrm{m}}, \omega_{\mathrm{f}}\right)$ is produced by left and right matrix multiplication of $\left[\widetilde{\mathbf{R}}_{\mathrm{g}}\left(\omega_{\mathrm{m}}, \omega_{\mathrm{s}}\right)\right]^{-1}$ with $\left[\mathbf{T}_{\mathrm{g}}\right]^{\mathrm{T}}$ and $\mathbf{T}_{\mathrm{g}}$ respectively.

After the stiffness matrix of the system has been obtained, the vehicle and the FE structures can be also added to the global system. The governing equation for partial coupling in the mixed FOR becomes: 


$$
\widetilde{\mathbf{K}}\left(\omega_{\mathrm{m}}, \omega_{\mathrm{f}}\right) \widetilde{\mathbf{U}}\left(\omega_{\mathrm{m}}, \omega_{\mathrm{f}}\right)=\tilde{\mathbf{F}}\left(\omega_{\mathrm{m}}, \omega_{\mathrm{f}}\right),
$$

where

$$
\begin{aligned}
& \widetilde{\mathbf{K}}\left(\omega_{\mathrm{m}}, \omega_{\mathrm{f}}\right)=\left[\begin{array}{ccccc}
\mathbf{K}_{\mathrm{v}}^{\mathrm{uu}}\left(\omega_{\mathrm{m}}\right) & \mathbf{K}_{\mathrm{v}}^{\mathrm{uw}}\left(\omega_{\mathrm{m}}\right) & \mathbf{0} & \mathbf{0} & \mathbf{0} \\
\mathbf{K}_{\mathrm{v}}^{\mathrm{wu}}\left(\omega_{\mathrm{m}}\right) & \mathbf{K}_{\mathrm{v}}^{\mathrm{ww}}\left(\omega_{\mathrm{m}}\right)+\mathbf{K}_{\mathrm{H}} & -\mathbf{K}_{\mathrm{H}} & \mathbf{0} & \mathbf{0} \\
\mathbf{0} & -\mathbf{K}_{\mathrm{H}} & \widetilde{\mathbf{K}}_{\mathrm{g}}^{\mathrm{rr}}\left(\omega_{\mathrm{m}}, \omega_{\mathrm{f}}\right)+\mathbf{K}_{\mathrm{H}} & \widetilde{\mathbf{K}}_{\mathrm{g}}^{\mathrm{rs}}\left(\omega_{\mathrm{f}}, \omega_{\mathrm{m}}\right) & \mathbf{0} \\
\mathbf{0} & \mathbf{0} & \widetilde{\mathbf{K}}_{\mathrm{g}}^{\mathrm{sr}}\left(\omega_{\mathrm{m}}, \omega_{\mathrm{f}}\right) & \widetilde{\mathbf{K}}_{\mathrm{g}}^{\mathrm{ss}}\left(\omega_{\mathrm{m}}, \omega_{\mathrm{f}}\right)+\widetilde{\mathbf{K}}_{\mathrm{FE}}^{\mathrm{ss}}\left(\omega_{\mathrm{f}}\right) \mathbf{K}_{\mathrm{FE}}^{\mathrm{sn}}\left(\omega_{\mathrm{f}}\right) \\
\mathbf{0} & \mathbf{0} & \mathbf{0} & \mathbf{K}_{\mathrm{FE}}^{\mathrm{ns}}\left(\omega_{\mathrm{f}}\right) & \mathbf{K}_{\mathrm{FE}}^{\mathrm{nn}}\left(\omega_{\mathrm{f}}\right)
\end{array}\right], \\
& \widetilde{\mathbf{U}}\left(\omega_{\mathrm{m}}, \omega_{\mathrm{f}}\right)=\left\{\begin{array}{c}
\widetilde{\mathbf{U}}_{\mathrm{v}}^{\mathrm{u}}\left(\omega_{\mathrm{m}}, \omega_{\mathrm{f}}\right) \\
\widetilde{\mathbf{U}}_{\mathrm{v}}^{\mathrm{w}}\left(\omega_{\mathrm{m}}, \omega_{\mathrm{f}}\right) \\
\widetilde{\mathbf{U}}_{\mathrm{g}}^{\mathrm{r}}\left(\omega_{\mathrm{m}}, \omega_{\mathrm{f}}\right) \\
\widetilde{\mathbf{U}}_{\mathrm{g}}^{\mathrm{s}}\left(\omega_{\mathrm{m}}, \omega_{\mathrm{f}}\right) \\
\widetilde{\mathbf{U}}_{\mathrm{FE}}^{\mathrm{n}}\left(\omega_{\mathrm{m}}, \omega_{\mathrm{f}}\right)
\end{array}\right\}, \quad \tilde{\mathbf{F}}\left(\omega_{\mathrm{m}}, \omega_{\mathrm{f}}\right)=\left\{\begin{array}{c}
\mathbf{0} \\
K_{\mathrm{H}} \mathbf{d}\left(\omega_{\mathrm{m}}\right) \\
-K_{\mathrm{H}} \mathbf{d}\left(\omega_{\mathrm{m}}\right) \\
\mathbf{0} \\
\mathbf{0}
\end{array}\right\}
\end{aligned}
$$

The superscripts relate to the degrees of freedom of: ' $w$ '—wheels, 'r'—rails, 's' — soil in the fixed FOR, ' $n$ '一 parts of FE structures not coupled to the soil and ' $u$ '—vehicles uncoupled from the track (e.g. the vehicle body). Vectors $\widetilde{\mathbf{U}}_{\mathrm{v}}, \widetilde{\mathbf{U}}_{\mathrm{g}}$ and $\widetilde{\mathbf{U}}_{\mathrm{FE}}$ store the displacements for the vehicle, global railway track-soil system and the FE structure, respectively. When the excitation frequency $\omega_{\mathrm{m}}$ is equal to zero, the unevenness of the track does not excite the system. In that case, the loads corresponding to the deadweight of the vehicle are placed directly on the rails.

Using the obtained formulation in Eq. (21) the system is solved for all combinations of moving $\omega_{\mathrm{m}}$ and fixed $\omega_{\mathrm{f}}$ FOR frequency pairs. The solution for each frequency pair is assumed to be independent of other pairs, and can be solved separately. Thus, the computational requirements for the system are greatly reduced. As the frequency pairs are not solved simultaneously some coupling effects through the frequencies are lost, making the system only partly coupled. The coupling between the two frames of reference introduced via Eqs. (17)(21) only couples the two investigated frequencies and does not include further spreading through the frequencies. For the investigated case, this means that the effects from the waves propagating through the soil and being reradiated by rigid bodies or FE structures will only affect the vehicle at the frequency of excitation and will not spread to a wider range of frequencies. In order to account for the coupling due to the Doppler effect observed in the moving FOR, related to reradiated waves from footings, etc., in the fixed FOR, and vice versa, the problem must be solved simultaneously for a number of combinations of the frequencies $\omega_{\mathrm{f}}$ and $\omega_{\mathrm{m}}$. This results in a system of equations that can be solved directly (see the next section) or by iteration.

It can be seen that the whole system can be solved as a single-step solution without the need of computing the wheel-rail interaction forces before applying them to the system. This way, the weak coupling between the vehicle and external structures coupled via the soil are also included in the system. For most practical applications, these effects are very small. However, the increase in computational effort needed to compute the full system is also relatively low, as the added vehicle degrees of freedom do not increase the total size of the matrix by much.

After the system has been solved, the displacement for the observation degrees of freedom in the fixed FOR can be obtained. For that purpose, a receptance matrix $\widetilde{\mathbf{R}}_{\text {or }}\left(\omega_{\mathrm{m}}, \omega_{\mathrm{f}}\right)$ between the rails and the observation nodes is established. The procedure follows Eq. (17) and the receptance matrix between the observation nodes and global nodes is constructed as:

$$
\widetilde{\mathbf{R}}_{\mathrm{og}}\left(\omega_{\mathrm{m}}, \omega_{\mathrm{f}}\right)=\left[\widetilde{\mathbf{R}}_{\mathrm{or}}\left(\omega_{\mathrm{m}}, \omega_{\mathrm{f}}\right) \quad \mathbf{R}_{\mathrm{os}}\left(\omega_{\mathrm{f}}\right)\right] .
$$

The displacements can then be obtained as 


$$
\widetilde{\mathbf{U}}_{\mathrm{o}}\left(\omega_{\mathrm{m}}, \omega_{\mathrm{f}}\right)=\widetilde{\mathbf{R}}_{\mathrm{og}}\left(\omega_{\mathrm{m}}, \omega_{\mathrm{f}}\right)\left[\widetilde{\mathbf{R}}_{\mathrm{gg}}\left(\omega_{\mathrm{m}}, \omega_{\mathrm{f}}\right)\right]^{-1} \mathbf{T}_{\mathrm{g}} \widetilde{\mathbf{U}}_{\mathrm{g}}\left(\omega_{\mathrm{m}}, \omega_{\mathrm{f}}\right)
$$

When the displacements for both the global and the observation degrees of freedom have been obtained, the effects from $J_{\mathrm{m}}$ discrete excitation frequencies $\omega_{\mathrm{m}}$ can be added together to obtain the total response in the fixed FOR:

$$
\mathbf{U}_{\mathrm{FE}}\left(\omega_{\mathrm{f}}\right)=\frac{1}{2 \pi} \sum_{j=1}^{J_{\mathrm{m}}} \widetilde{\mathbf{U}}_{\mathrm{FE}}\left(\omega_{\mathrm{m}, j}, \omega_{\mathrm{f}}\right) D\left(k_{\mathrm{m}, j}\right) \Delta k_{\mathrm{m}},
$$

where $D\left(k_{\mathrm{m}, j}\right)$ is the rail unevenness obtained from a power spectral density (PSD) according to the wavenumber $k_{\mathrm{m}, j}$, and $\Delta k_{\mathrm{m}}$ is the wavenumber step size. The wavenumber can be found as

$$
k_{\mathrm{m}, j}=\frac{\omega_{\mathrm{m}, j}}{v} .
$$

The displacement vectors $\widetilde{\mathbf{U}}_{\mathrm{g}}^{\mathrm{s}}$ for the global-system soil displacements and $\widetilde{\mathbf{U}}_{\mathrm{o}}$ for the observation node displacements, both relating to the fixed FOR, can be constructed using the same approach. Meanwhile, the displacement vectors $\widetilde{\mathbf{U}}_{\mathrm{g}}^{\mathrm{r}}$ for the global-system rail displacements and $\widetilde{\mathbf{U}}_{\mathrm{v}}$ for the vehicle displacements both relate to the moving FOR. Hence, they should be constructed by combining the effects of the entire considered range of frequencies $\omega_{\mathrm{f}}$ in the fixed FOR.

Further, the time-domain response for the degrees of freedom associated with the fixed FOR can be obtained by performing an inverse discrete Fourier transformation of the displacements $\mathbf{U}_{\mathrm{FE}}\left(\omega_{\mathrm{s}}\right)$ :

$$
\mathbf{u}_{\mathrm{FE}}(t)=\frac{1}{2 \pi} \sum_{j=1}^{J_{\mathrm{f}}} \mathbf{U}_{\mathrm{FE}}\left(\omega_{\mathrm{f}, j}\right) \mathrm{e}^{\mathrm{i} \omega_{\mathrm{f}, j} t} \Delta \omega_{\mathrm{f}},
$$

where $J_{\mathrm{f}}$ is the number of discrete frequencies in the fixed FOR. Note that $J_{\mathrm{f}}$ and $J_{\mathrm{m}}$ need not be equal. However, the step sizes $\Delta \omega_{\mathrm{f}}$ and $\Delta k_{\mathrm{m}}$ must be small enough, and the number of frequencies $J_{\mathrm{f}}$ and $J_{\mathrm{m}}$ large enough, to ensure proper discretization of peaks in the loads and resonances of the system while, at the same time, avoiding violation of the periodicity inherent in the frequency-domain solution. Thus, analysis of trains passing very slowly over a track with both short and long irregularities is computationally demanding.

The methodology presented here can be easily expanded for a wider variety of cases. Using the established coupling between two frames of reference, it is also possible to add FE structures in the moving FOR in order to model a more complex geometry of the track or vehicle. Further, the receptance matrices can be created with other methods, for example FE or BE methods, with the global system being assembled and solved in the same manner. Finally, it is noted that the methodology is well-suited for calculation on any computer system, ranging from laptops to large clusters, given that parallelization is possible at many levels.

\subsection{Fully coupled global system}

Using the matrices already created for the previously described solution procedure, it is also possible to assemble a fully coupled global system. Such a system accounts for the frequency spreading of the reflected waves due to the Doppler effect and might be useful for certain cases where the full coupling between the vehicle (the source) and the structures (the receivers) cannot be discarded. Problems where these effects are important could include modelling of tunnels underneath buildings, railway stations and various structures nearby the tracks. Several such cases have been investigated by Coulier et al. [40], finding that while the axial loads are not effected significantly, the vibration insertion gain for source-receiver transmission can be affected up to $10 \mathrm{~dB}$, when using a fully coupled solution procedure. Another set of problems could include the sudden change of stiffness underneath the track, e.g. when a concreate passage is constructed underneath 
the railway track. Similar problem is investigated in Section 6.2 modelling a rigid block buried below the track using a fully coupled approach.

Considering a discretized system with $J_{\mathrm{f}}$ frequencies in the fixed FOR and $J_{\mathrm{m}}$ frequencies in the moving FOR, the global receptance matrix for the fully coupled system is assembled as:

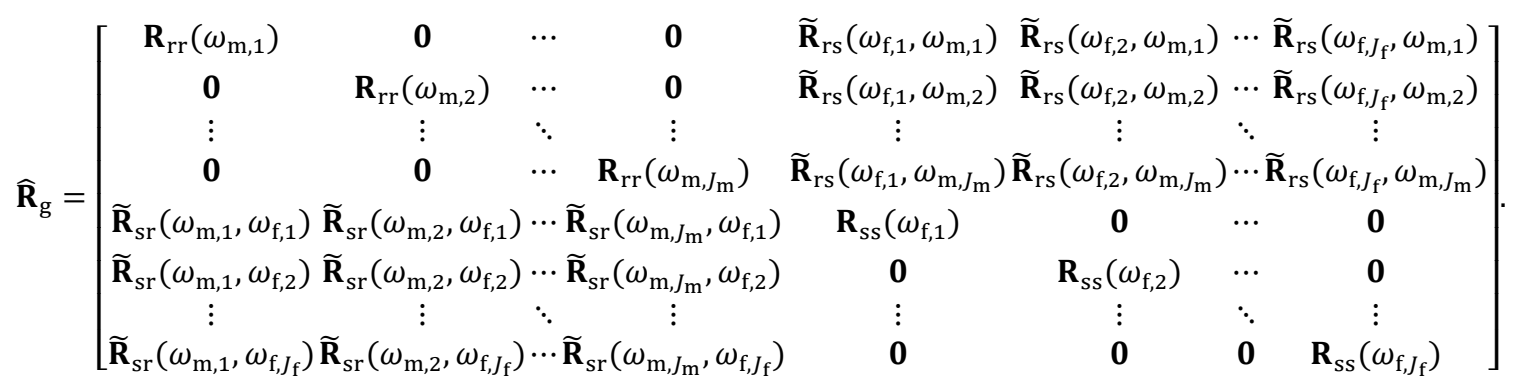

The overhat notation on $\widehat{\mathbf{R}}_{\mathrm{g}}$ indicates the two-way coupling, and again subscript ' $\mathrm{g}$ ' stands for 'global'. The matrices that depend on single frequencies are assembled into a block diagonal matrices, while the coupling terms that depend on pairs of frequencies are fully populated submatrices. It can be seen that the previously uncoupled frequencies in a single FOR are now coupled through the other FOR. To consider rigid objects, the transformation matrix for the considered system is also needed. However, the transformation matrices are frequency independent and can be assembled simply by combining the previously obtained matrices into a block diagonal matrix, expanding Eq. (20) into

$$
\widehat{\mathbf{T}}_{\mathrm{g}}=\operatorname{diag}\left\{\begin{array}{llllllll}
\mathbf{I}_{1} & \mathbf{I}_{2} & \cdots & \mathbf{I}_{J_{\mathrm{m}}} & \mathbf{T}_{\mathrm{s}, 1} & \mathbf{T}_{\mathrm{s}, 2} & \cdots & \mathbf{T}_{\mathrm{s}, J_{\mathrm{f}}},
\end{array}\right\},
$$

where in total there are $J_{\mathrm{m}}$ identity matrices, $\mathbf{I}$, and $J_{\mathrm{f}}$ local transformation matrices, $\mathbf{T}_{\mathrm{s}}$. To obtain the stiffness matrix, the system is solved in the same manner as in the partly coupled system:

$$
\widehat{\mathbf{K}}_{\mathrm{g}}=\left[\widehat{\mathbf{T}}_{\mathrm{g}}\right]^{\mathrm{T}}\left[\widehat{\mathbf{R}}_{\mathrm{g}}\right]^{-1} \widehat{\mathbf{T}}_{\mathrm{g}} \text {. }
$$

In most cases, the matrix $\widehat{\mathbf{K}}_{\mathrm{g}}$ will be fully populated. To add the vehicle and external FE structures to the system, the fully coupled stiffness matrix $\widehat{\mathbf{K}}_{\mathrm{g}}$ is split into submatrices: $\widehat{\mathbf{K}}_{\mathrm{g}}^{\mathrm{rr}, j_{\mathrm{m}} j_{\mathrm{m}}}\left(\omega_{\mathrm{m}, j_{\mathrm{m}}}\right)$ corresponding to the rail degrees of freedom in a moving FOR at the discrete frequency $\omega_{\mathrm{m}, j_{\mathrm{m}}}, \widehat{\mathbf{K}}_{\mathrm{g}}^{\mathrm{ss}, j_{\mathrm{f}} j_{\mathrm{f}}}\left(\omega_{\mathrm{f}, j_{\mathrm{f}}}\right)$ corresponding to the soil degrees of freedom at a discrete frequency $\omega_{\mathrm{f}, j_{\mathrm{f}}}$ in the fixed FOR. Further, coupling terms between the moving FOR rails and fixed FOR soil are $\widehat{\mathbf{K}}_{\mathrm{g}}^{\mathrm{rs}, j_{\mathrm{m}} j_{\mathrm{f}}}\left(\omega_{\mathrm{m}, j_{\mathrm{m}}}, \omega_{\mathrm{f}, j_{\mathrm{f}}}\right)$ and $\widehat{\mathbf{K}}_{\mathrm{g}}^{\mathrm{sr}, j_{\mathrm{f}} j_{\mathrm{m}}}\left(\omega_{\mathrm{f}, j_{\mathrm{f}}}, \omega_{\mathrm{m}, j_{\mathrm{m}}}\right)$. Differently from the partly coupled system, the fully coupled system has terms coupling the soil degrees of freedom at one fixed-FOR frequency $\omega_{\mathrm{f}, j_{\mathrm{f}}}$ to the soil degrees of freedom at another fixed-FOR frequency $\omega_{\mathrm{f}, i_{\mathrm{f}}}$, such as $\widehat{\mathbf{K}}_{\mathrm{g}}^{\mathrm{s}, j_{\mathrm{f}} i_{\mathrm{f}}}\left(\omega_{\mathrm{f}, j_{\mathrm{f}}}, \omega_{\mathrm{f}, i_{\mathrm{f}}}\right)$ and $\widehat{\mathbf{K}}_{\mathrm{g}}^{\mathrm{ss}, i_{\mathrm{f}} j_{\mathrm{f}}}\left(\omega_{\mathrm{f}, i_{\mathrm{f}}}, \omega_{\mathrm{f}, j_{\mathrm{f}}}\right)$. Similarly, the rail degrees of freedom are coupled between $\omega_{\mathrm{m}, j_{\mathrm{f}}}$ and $\omega_{\mathrm{m}, i_{\mathrm{f}}}$ in the moving FOR through $\widehat{\mathbf{K}}_{\mathrm{g}}^{\mathrm{rr}, j_{\mathrm{m}} i_{\mathrm{m}}}\left(\omega_{\mathrm{m}, j_{\mathrm{m}}}, \omega_{\mathrm{m}, i_{\mathrm{m}}}\right)$ and $\widehat{\mathbf{K}}_{\mathrm{g}}^{\mathrm{rr}, i_{\mathrm{m}} j_{\mathrm{m}}}\left(\omega_{\mathrm{m}, i_{\mathrm{m}}}, \omega_{\mathrm{m}, j_{\mathrm{m}}}\right)$.

Using the submatrices defined above, the vehicle is coupled to the rails at each moving-FOR frequency $\omega_{\mathrm{m}, j_{\mathrm{m}}}$ :

$$
\widehat{\mathbf{K}}_{\mathrm{mm}}^{j_{\mathrm{m}} j_{\mathrm{m}}}\left(\omega_{\mathrm{m}, j_{\mathrm{m}}}\right)=\left[\begin{array}{ccc}
\mathbf{K}_{\mathrm{v}}^{\mathrm{uu}}\left(\omega_{\mathrm{m}, j_{\mathrm{m}}}\right) & \mathbf{K}_{\mathrm{v}}^{\mathrm{uw}}\left(\omega_{\mathrm{m}, j_{\mathrm{m}}}\right) & \mathbf{0} \\
\mathbf{K}_{\mathrm{v}}^{\mathrm{wu}}\left(\omega_{\mathrm{m}, j_{\mathrm{m}}}\right) & \mathbf{K}_{\mathrm{v}}^{\mathrm{ww}}\left(\omega_{\mathrm{m}, j_{\mathrm{m}}}\right)+\mathbf{K}_{\mathrm{H}} & -\mathbf{K}_{\mathrm{H}} \\
\mathbf{0} & -\mathbf{K}_{\mathrm{H}} & \widehat{\mathbf{K}}_{\mathrm{g}}^{\mathrm{rr}, j_{\mathrm{m}} j_{\mathrm{m}}}\left(\omega_{\mathrm{m}, j_{\mathrm{m}}}\right)+\mathbf{K}_{\mathrm{H}}
\end{array}\right]
$$

with subscript ' $\mathrm{m}$ ' referring to all degrees of freedom in the moving FOR, i.e. the combination of the uncoupled vehicle ' $u$ ', wheel ' $w$ ' and rail ' $r$ ' degrees of freedom. The force matrix from the track unevenness is also created: 


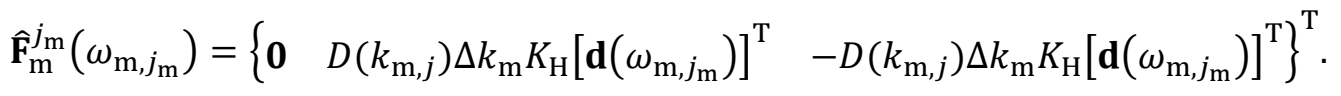

Note that the track unevenness $D$ has to be inserted in the force matrix here, as the obtained displacements will only depend on a single frequency. If the moving-FOR frequency $\omega_{\mathrm{m}, j_{\mathrm{m}}}$ is equal to zero, the deadweight of the vehicle is applied directly to the rails. However, the vehicle is still added to the system, since it reacts to the reradiated waves propagating back into the moving-FOR system from structures defined in the fixed FOR.

External FE structures can be coupled to the soil at fixed FOR frequency $\omega_{\mathrm{f}, j_{\mathrm{f}}}$ :

$$
\widehat{\mathbf{K}}_{\mathrm{ff}}^{j_{\mathrm{f}} j_{\mathrm{f}}}\left(\omega_{\mathrm{f}, j_{\mathrm{f}}}\right)=\left[\begin{array}{cc}
\widehat{\mathbf{K}}_{\mathrm{g}}^{\mathrm{ss}, j_{\mathrm{f}} j_{\mathrm{f}}}\left(\omega_{\mathrm{f}, j_{\mathrm{f}}}\right)+\mathbf{K}_{\mathrm{FE}}^{\mathrm{ss}}\left(\omega_{\mathrm{f}, j_{\mathrm{f}}}\right) & \mathbf{K}_{\mathrm{FE}}^{\mathrm{sn}}\left(\omega_{\mathrm{f}, j_{\mathrm{f}}}\right) \\
\mathbf{K}_{\mathrm{FE}}^{\mathrm{ns}}\left(\omega_{\mathrm{f}, j_{\mathrm{f}}}\right) & \mathbf{K}_{\mathrm{FE}}^{\mathrm{nn}}\left(\omega_{\mathrm{f}, j_{\mathrm{f}}}\right)
\end{array}\right],
$$

here the subscript ' $\mathrm{f}$ ' refers to all degrees of freedom in a fixed FOR in the full system, it is a combination of soil 's' and uncoupled FE ' $u$ ' degrees of freedom. In this case they are all related to the building structure. Further, the coupling terms coupling the system through frequencies also need to be included. Nothing is directly coupled to them, thus only zero terms are added to the matrix to ensure the obtained matrices are sized correctly. For example, to include the coupling term $\widehat{\mathbf{K}}_{\mathrm{g}}^{\mathrm{rs}, j_{\mathrm{f}} j_{\mathrm{m}}}$ in the full system:

$$
\widehat{\mathbf{K}}_{\mathrm{mf}}^{j_{\mathrm{m}} j_{\mathrm{f}}}\left(\omega_{\mathrm{m}, j_{\mathrm{m}}}, \omega_{\mathrm{f}, j_{\mathrm{f}}}\right)=\left[\begin{array}{cc}
\mathbf{0}^{(\mathrm{uw} \times \mathrm{s})} & \mathbf{0}^{(\mathrm{uw} \times \mathrm{n})} \\
\widehat{\mathbf{K}}_{\mathrm{g}}^{\mathrm{rs}, j_{\mathrm{m}} j_{\mathrm{f}}}\left(\omega_{\mathrm{m}, j_{\mathrm{m}}}, \omega_{\mathrm{f}, j_{\mathrm{f}}}\right) & \mathbf{0}^{(\mathrm{r} \times \mathrm{n})}
\end{array}\right],
$$

where the superscripts of the zero matrices indicate the sizes of the submatrices, e.g. uw $\times \mathrm{n}$ indicates that the submatrix has a number of rows equal to the number of degrees of freedom in the vehicle (combined uncoupled and wheel degrees of freedom) and a number of columns equal to the number of degrees of freedom of not coupled FE structure. Similarly, the matrix coupling the soil degrees of freedom between two frequencies is defined as

$$
\widehat{\mathbf{K}}_{\mathrm{ff}}^{j_{\mathrm{f}} i_{\mathrm{f}}}\left(\omega_{\mathrm{f}, j_{\mathrm{f}}}, \omega_{\mathrm{f}, i_{\mathrm{f}}}\right)=\left[\begin{array}{cc}
\widehat{\mathbf{K}}_{\mathrm{g}}^{\mathrm{ss}, j_{\mathrm{f}} i_{\mathrm{f}}}\left(\omega_{\mathrm{f}, j_{\mathrm{f}}}, \omega_{\mathrm{f}, i_{\mathrm{f}}}\right) & \mathbf{0}^{(\mathrm{s} \times \mathrm{n})} \\
\mathbf{0}^{(\mathrm{n} \times \mathrm{s})} & \mathbf{0}^{(\mathrm{n} \times \mathrm{n})}
\end{array}\right] .
$$

This is repeated to create the other coupling matrices in the full system, i.e. $\widehat{\mathbf{K}}_{\mathrm{fm}}^{j_{\mathrm{f}} j_{\mathrm{m}}}\left(\omega_{\mathrm{f}, j_{\mathrm{f}}}, \omega_{\mathrm{m}, j_{\mathrm{m}}}\right)$, $\widehat{\mathbf{K}}_{\mathrm{ff}}^{i_{\mathrm{f}} j_{\mathrm{f}}}\left(\omega_{\mathrm{f}, i_{\mathrm{f}}}, \omega_{\mathrm{f}, j_{\mathrm{f}}}\right), \widehat{\mathbf{K}}_{\mathrm{mm}}^{j_{\mathrm{m}} i_{\mathrm{m}}}\left(\omega_{\mathrm{m}, j_{\mathrm{m}}}, \omega_{\mathrm{m}, i_{\mathrm{m}}}\right)$ and $\widehat{\mathbf{K}}_{\mathrm{mm}}^{i_{\mathrm{m}} j_{\mathrm{m}}}\left(\omega_{\mathrm{m}, i_{\mathrm{m}}}, \omega_{\mathrm{m}, j_{\mathrm{m}}}\right)$. These are created from the global system matrices $\widehat{\mathbf{K}}_{\mathrm{g}}^{\mathrm{r}, j_{\mathrm{f}} j_{\mathrm{m}}}\left(\omega_{\mathrm{f}, j_{\mathrm{f}}}, \omega_{\mathrm{m}, j_{\mathrm{m}}}\right), \widehat{\mathbf{K}}_{\mathrm{g}}^{\mathrm{ss}, i_{\mathrm{f}} j_{\mathrm{f}}}\left(\omega_{\mathrm{f}, i_{\mathrm{f}}}, \omega_{\mathrm{f}, j_{\mathrm{f}}}\right), \widehat{\mathbf{K}}_{\mathrm{g}}^{\mathrm{rr}, j_{\mathrm{m}} i_{\mathrm{m}}}\left(\omega_{\mathrm{m}, j_{\mathrm{m}}}, \omega_{\mathrm{m}, i_{\mathrm{m}}}\right)$ and $\widehat{\mathbf{K}}_{\mathrm{g}}^{\mathrm{rr}, i_{\mathrm{m}} j_{\mathrm{m}}}\left(\omega_{\mathrm{m}, i_{\mathrm{m}}}, \omega_{\mathrm{m}, j_{\mathrm{m}}}\right)$, respectively.

The full system is assembled into submatrices related to the moving and fixed FORs and the coupling terms between them:

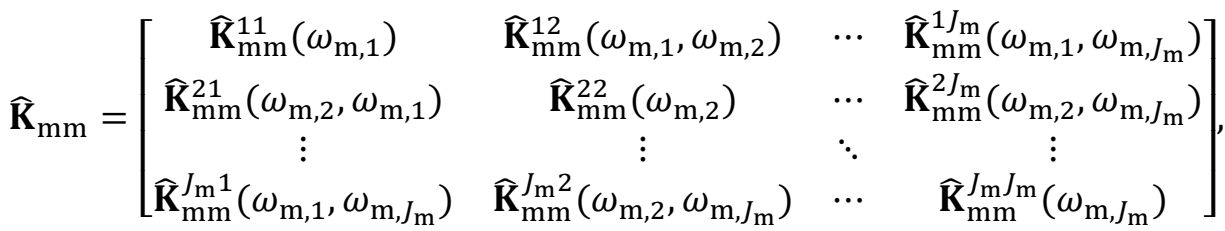

$$
\begin{aligned}
& \widehat{\mathbf{K}}_{\mathrm{ff}}=\left[\begin{array}{cccc}
\widehat{\mathbf{K}}_{\mathrm{ff}}^{11}\left(\omega_{\mathrm{f}, 1}\right) & \widehat{\mathbf{K}}_{\mathrm{ff}}^{12}\left(\omega_{\mathrm{f}, 1}, \omega_{\mathrm{f}, 2}\right) & \cdots & \widehat{\mathbf{K}}_{\mathrm{ff}}^{1 J_{\mathrm{f}}}\left(\omega_{\mathrm{f}, 1}, \omega_{\mathrm{f}, J_{\mathrm{f}}}\right) \\
\widehat{\mathbf{K}}_{\mathrm{ff}}^{21}\left(\omega_{\mathrm{f}, 2}, \omega_{\mathrm{f}, 1}\right) & \widehat{\mathbf{K}}_{\mathrm{ff}}^{22}\left(\omega_{\mathrm{f}, 2}\right) & \cdots & \widehat{\mathbf{K}}_{\mathrm{ff}}^{2 J_{\mathrm{f}}}\left(\omega_{\mathrm{f}, 2}, \omega_{\mathrm{f}, J_{\mathrm{f}}}\right) \\
\vdots & \vdots & \ddots & \vdots \\
\widehat{\mathbf{K}}_{\mathrm{ff}}^{J_{\mathrm{f}} 1}\left(\omega_{\mathrm{f}, J_{\mathrm{f}}}, \omega_{\mathrm{f}, 1}\right) & \widehat{\mathbf{K}}_{\mathrm{ff}}^{J_{\mathrm{f}} 2}\left(\omega_{\mathrm{f}, J_{\mathrm{f}}}, \omega_{\mathrm{f}, 2}\right) & \cdots & \widehat{\mathbf{K}}_{\mathrm{ff}}^{J_{\mathrm{ff}} J_{\mathrm{f}}}\left(\omega_{\mathrm{f}, J_{\mathrm{f}}}\right)
\end{array}\right],
\end{aligned}
$$




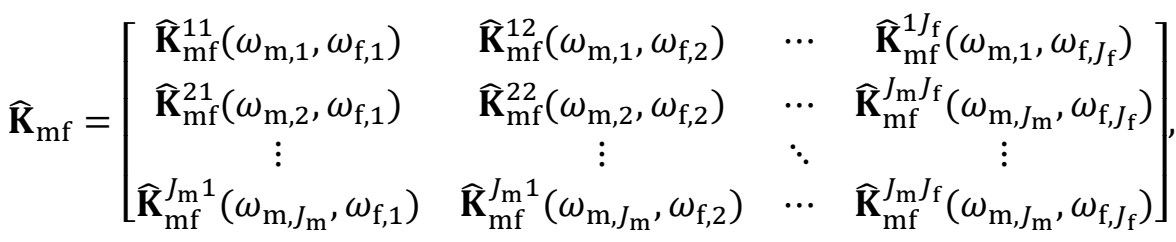

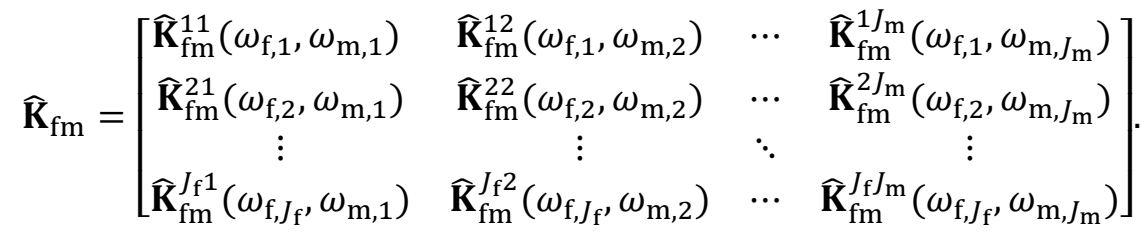

Next, the full system displacement and load vectors are created:

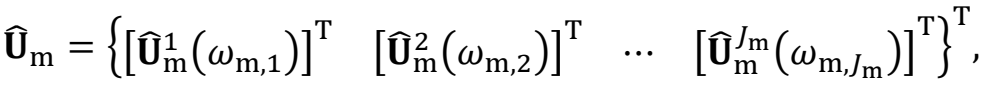

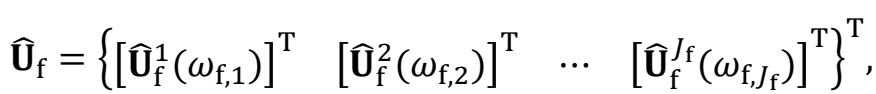

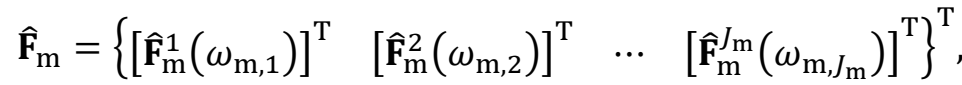

where it is assumed that only load in a moving FOR is considered. The full system can be solved to obtain the displacements as:

$$
\widehat{\mathbf{K}} \widehat{\mathbf{U}}=\widehat{\mathbf{F}}, \quad \overleftrightarrow{\mathbf{K}}=\left[\begin{array}{cc}
\widehat{\mathbf{K}}_{\mathrm{mm}} & \widehat{\mathbf{K}}_{\mathrm{mf}} \\
\widehat{\mathbf{K}}_{\mathrm{fm}} & \widehat{\mathbf{K}}_{\mathrm{ff}}
\end{array}\right], \quad \widehat{\mathbf{U}}=\left\{\begin{array}{c}
\widehat{\mathbf{U}}_{\mathrm{m}} \\
\widehat{\mathbf{U}}_{\mathrm{f}}
\end{array}\right\}, \quad \widehat{\mathbf{F}}=\left\{\begin{array}{c}
\widehat{\mathbf{F}}_{\mathrm{m}} \\
\widehat{\mathbf{F}}_{\mathrm{f}}
\end{array}\right\} .
$$

In this case, the vector $\widehat{\mathbf{F}}_{\mathrm{f}}$ is all zeros as there are no loads acting in the fixed FOR. However, the system can be solved by applying loads in either FOR or both FORs at the same time. After the displacements have been obtained, the time-domain solution for both FORs can be obtained using Eq. (26). To obtain the displacements of observation nodes, a receptance matrix equivalent to the two lower quarters of the matrix in Eq. (27) should be constructed, relating the observation nodes to the rails and to the soil. However, for any considerable number of observation degrees of freedom, the matrix size becomes very large, which can be considered a computational drawback of the methodology.

It is evident that the computation of the fully coupled system is an extremely computationally demanding process, which involves double inversion of very large matrices. Thus, it should only be used when the secondary coupling effects are an important factor. The fully coupled system computation greatly depends on the random-access memory (RAM) available in the computer system used, as the matrices are generally fully populated. However, if the considered system is not too large and the matrices can be stored in computer RAM, the computation times for the system are almost equal to those the partly coupled system. The efficiency of the solution procedure could be expanded by limiting the range through which the frequencies can interact between the two FORs.

\section{Validation}

The methodology described in this paper is validated by comparison with other computational approaches. Firstly, considering a system with no structures or rigid objects interacting with the soil, the described system assembly and solution procedures provide identical results to the approach provided by Sheng et al. [10-12]. This is expected, as this work utilizes the same vehicle-track-soil interaction model, and, with no other structures interacting with the soil, the coupling terms cancel out. Further, modelling of rigid objects interacting with the soil was validated by comparison with BE and FEM-PML models. It was determined that the semianalytical model provides a very good match, especially with the FEM-PML model, where even the secondary 
coupling terms show very good agreement. Sections 5.1 and 5.2 provide a more in-depth descriptions of validating the coupling terms used in the system assembly and the solution procedure including a structure interacting with the soil.

\subsection{Coupling between moving and fixed frames of reference}

In order to validate the coupling terms between the two frames of reference, especially the symmetry between a load applied in the moving FOR and the displacements observed in a fixed FOR and vice versa, some test cases were set up. The previously described mixed-FOR model was simplified by removing the railway track together with the vehicle and the FE/rigid structures interacting with the soil. This way, the effects of a single load with a single excitation frequency acting directly on the soil surface can be observed. The ground was modelled as a homogenous elastic half-space of dense sandy-type soil, with a Young's modulus of $250 \mathrm{MPa}$, a Poisson's ratio of 0.25 , a mass density of $2000 \mathrm{~kg} / \mathrm{m}^{3}$ and a loss factor of 0.05 . A stationary point in the fixed FOR was placed $3 \mathrm{~m}$ from the line along which the load was moving.

Firstly, a moving vertical load with constant speed $v$ and frequency $f_{\mathrm{m}}$ was modelled. The vertical displacements were observed within a fixed FOR for a range of frequencies $f_{\mathrm{f}}$. The system was modelled using the simplified mixed-FOR model. For the analysed case, only the coupling terms between the moving and the fixed frames of reference, as described in Section 2.2, have an effect for the obtained results. For comparison, a full model was established, modelled only in the moving FOR, using the semi-analytical approach.

Using the created model, the displacement field of the soil surface in the moving FOR and within the time domain was obtained for a single excitation frequency. Then, a time signal for displacements of an observation point moving through the displacement field with speed $-v$ was found, considering that for every time step the position of the observation point changed. Fourier transforming the obtained time signal into frequencies $f_{s}$, the displacement spectra for a stationary observation point was obtained. Two combinations of speed and frequency in the moving FOR were investigated: Combination 1 with $v=20 \mathrm{~m} / \mathrm{s}$ and $f_{\mathrm{m}}=20 \mathrm{~Hz}$, and Combination 2 with $v=40 \mathrm{~m} / \mathrm{s}$ and $f_{\mathrm{m}}=10 \mathrm{~Hz}$. The comparison of both approaches is shown in Figure 1 where it can be seen that the two approaches agree, confirming the analytical derivation presented in Section 2.2.

Secondly, a similar test case was created to analyse the coupling between a load in a fixed FOR and the resulting displacements in a moving FOR. Here, the mixed FOR model was compared to a full solution formulated only in a fixed FOR. In the same way as for the previous case, using the full model, the displacement field of the soil surface was found in time domain. Then the vertical displacements for a moving observation point were obtained by changing the position of the point for every time step. Fourier transforming the time-domain response for frequencies $f_{\mathrm{m}}$, the displacement spectra for a moving observation point were
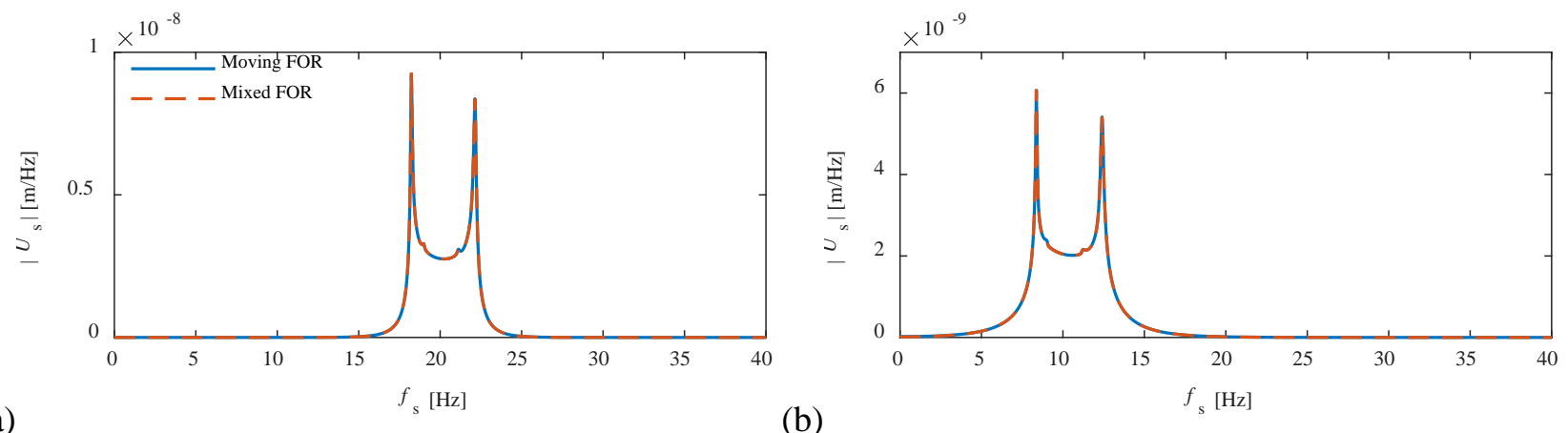

Figure 1. Displacements in a fixed FOR from a unit load applied in a moving FOR: (a) $v=20 \mathrm{~m} / \mathrm{s}$ and $f_{\mathrm{m}}=$ $20 \mathrm{~Hz}$; (b) $v=40 \mathrm{~m} / \mathrm{s}$ and $f_{\mathrm{m}}=10 \mathrm{~Hz}$. 


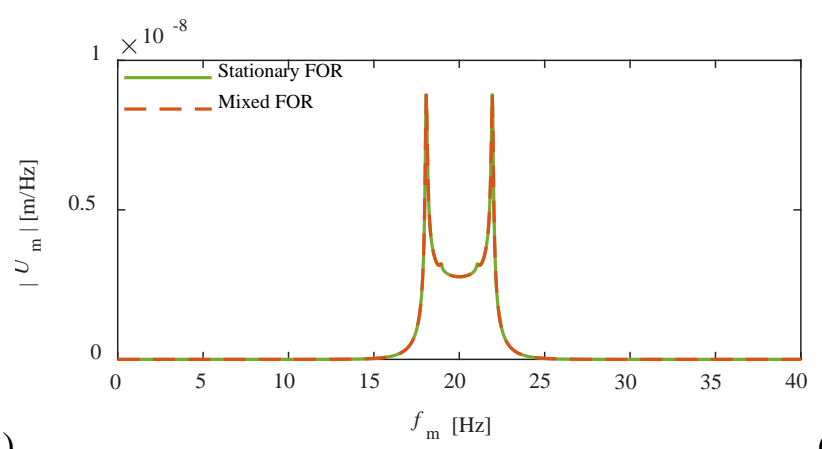

(a)

Figure 2. Displacements in a moving FOR from a unit load applied in a fixed FOR.: (a) $v=20 \mathrm{~m} / \mathrm{s}$ and $f_{\mathrm{s}}=$ $20 \mathrm{~Hz}$; (b) $v=40 \mathrm{~m} / \mathrm{s}$ and $f_{\mathrm{s}}=10 \mathrm{~Hz}$.

obtained. Two combinations of speed and stationary frequency were again investigated: Combination 1 with $v=20 \mathrm{~m} / \mathrm{s}$ and $f_{\mathrm{s}}=20 \mathrm{~Hz}$; Combination 2 with $v=40 \mathrm{~m} / \mathrm{s}$ and $f_{\mathrm{s}}=10 \mathrm{~Hz}$. Results of both approaches are given in Figure 2. Once again, the results agree well, this time confirming the derivation given in Section 2.3.

Comparing the two investigated cases, it is evident that the response spreads out through the observer frequencies due to the Doppler effect, independently of which FOR the load was applied in. The range of affected frequencies are dependent on the speed of the moving FOR. However, a load applied in the fixed FOR produces two symmetric peaks around the excitation frequency, when observed in a moving FOR, while a load applied in a moving FOR produces two peaks in the fixed FOR. Further, comparing the results of Combination 1 for both cases, it can be observed that the result in Figure 1 at $f_{\mathrm{s}}=20 \mathrm{~Hz}$ and the result in Figure 2 at $f_{\mathrm{m}}=20 \mathrm{~Hz}$ show exactly the same response. This confirms the symmetry between the two frames of reference implied by Eq. (7). The same result is obtained by comparing the responses of Combination 2 for both cases.

\subsection{Validation of the modelling approach}

To validate the modelling approach presented in this paper, it was compared to a sub-modelling technique, as described by [31]. A similar modelling approach was also used in [23]. In both cases the sub-modelling

Figure 3. Investigated case for validating the modelling approach. The vehicle is travelling from left to right, with magenta nodes indicating the wheel positions. The red nodes indicate the observation points, while the shades of colour indicate the vertical displacements (bright yellow is up, dark blue is down). Track unevenness is not scaled. 


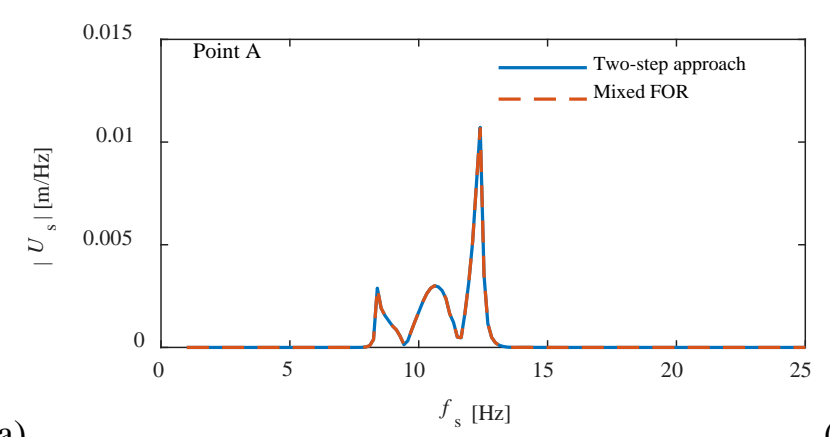

(a)

Figure 4. Comparison between two system solution procedures. Displacements are shown: (a) at the centre of the ground floor; (b) at the centre of the second floor of the building structure.

approach was used to model an underground railway tunnel with a vehicle travelling through it, in turn exciting a building structure above the soil. However, the solution procedure can also be applied to surface railways and the system analysed in this work. The method uses the free-field displacements of the soil caused by a passing vehicle, which are later modified by introducing the building structure. Ensuring equilibrium and compatibility between the degrees of freedom connecting the building and the soil, a solution for the building displacements can be found. Comparing the methodology proposed in this work with the sub-modelling technique, it becomes evident that the basic parts used for both solution procedures are identical. For example, the free-field displacements from a moving load used in the two-step approach are identical to the flexibility matrix $\mathbf{G}_{\mathrm{rs}}$ used here. However, the assembly and solution of the full system is somewhat different, with the proposed methodology allowing a wider range of applications, such as modelling rigid inclusions or allowing a two-way coupling between the two frames of reference. Further, the sub-modelling technique, as applied in this work, is a two-step solution approach, as the wheel-rail interaction forces are obtained in the moving FOR before being used to obtain the displacements in the fixed FOR.

As, both approaches use the same basic parts, it is relatively easy to compare them. For comparison, a building structure, as described below in Section 6.1, was used. However, the sub-modelling technique cannot directly model rigid objects, thus the rigid surface footings underneath each column were replaced by a single flexible slab footing underneath the whole building. The foundation slab was modelled using shell finite elements with the same properties as the building floors. The soil is modelled as a half-space of sand, with the same properties as in Section 5.1. Both systems were excited by a single passing vehicle, travelling at $40 \mathrm{~m} / \mathrm{s}$, exposed to a unit rail unevenness, with a $4 \mathrm{~m}$ wavelength corresponding to an excitation frequency of $10 \mathrm{~Hz}$. For analysis, only a single excitation frequency is used, as the effects from multiple excitations frequencies are just added together due to the principal of superposition. Thus, a single excitation frequency is enough to evaluate how well both models perform. The test case is illustrated in Figure 3.

Figure 4 shows the displacements of the building structure obtained at the centre of the ground floor and the second floor. It can be observed that both approaches provide almost identical results, showing that the proposed method is performing well. At the same time, almost identical results indicate that the back-coupling of the building structure to the railway track is insignificant. It can be concluded, that at least for the considered case, there is no significant difference whether on or the other approach is considered.

\section{Example cases}

\subsection{Partly coupled system}

To demonstrate the capabilities of the proposed partly-coupled-modelling approach, an example case was set up. A simple building structure that is excited by a vehicle travelling on a nearby railway track is modelled. The vehicle, railway track, soil and the building were all modelled in one coupled system. The soil was 
Table 1. Vehicle properties.

\begin{tabular}{|l|c|l|}
\hline Mass of car body & 40000 & $\mathrm{~kg}$ \\
\hline Mass of bogie & 5000 & $\mathrm{~kg}$ \\
\hline Mass of wheel set & 1800 & $\mathrm{~kg}$ \\
\hline Car body pitch moment of inertia & $2.0 \cdot 10^{6}$ & $\mathrm{~kg} \cdot \mathrm{m}^{2}$ \\
\hline Primary suspension stiffness & $2.4 \cdot 10^{6}$ & $\mathrm{~N} / \mathrm{m}$ \\
\hline Secondary suspension stiffness & $6.0 \cdot 10^{5}$ & $\mathrm{~N} / \mathrm{m}$ \\
\hline Primary suspension damping & 30000 & $\mathrm{~N} \cdot \mathrm{s} / \mathrm{m}$ \\
\hline Secondary suspension damping & 20000 & $\mathrm{~N} \cdot \mathrm{s} / \mathrm{m}$ \\
\hline Distance between bogies' centers & 19.0 & $\mathrm{~m}$ \\
\hline Distance between bogie's wheels sets & 2.7 & $\mathrm{~m}$ \\
\hline Herztian constant $G_{H}$ & $5.14 \cdot 10^{-8}$ & $\mathrm{~m} / \mathrm{N}^{2 / 3}$ \\
\hline
\end{tabular}

modelled as a $5 \mathrm{~m}$ layer of soft clay sitting over a stiffer half-space of sand. The clay had a Young's modulus of $80 \mathrm{MPa}$, a Poisson's ratio of 0.48 , a mass density of $2100 \mathrm{~kg} / \mathrm{m}^{3}$, and a loss factor of 0.05 . The underlying sand had the same properties as in Section 5. A soil stratification with two layers was chosen, as the waves reflecting from the interface between the two different materials can, in some cases, amplify the excitation of the building structure.

Only a single vehicle was modelled in the system. It was modelled as a 10-degree-of-freedom multibody system. The system consists of a rigid vehicle body, two rigid bogies and four wheels, with parts of the vehicle connected through a two-tier spring dashpot system. The vehicle was travelling at $40 \mathrm{~m} / \mathrm{s}$ over the track structure, with the building $10 \mathrm{~m}$ away from the track centreline. The properties of the vehicle and the track are given in Tables 1 and 2, respectively. The system was excited by the deadweight of the vehicle and by the dynamic wheel-rail interaction forces caused by the uneven vertical track profile. To model the unevenness of the rails, a German track spectrum was used to obtain the PSD function, as described by Cantero et al. [41], with the track quality coefficient equal to $0.59233 \cdot 10^{-6}$, corresponding to a medium quality track. From it, the corresponding unevenness values for every wavenumber were obtained. The track unevenness was considered in the range $0.5-80 \mathrm{~m}$, including both positive and negative wavenumbers to allow a double-sided Fourier transformation. The total amount of discrete wavenumbers considered was 800 .

The modelled building structure can be seen in Figure 5. It is a simple structure with six columns supporting two floors. Underneath each column, a square rigid surface footing was modelled, with one side equal to $2 \mathrm{~m}$. Each rigid footing was discretized into 100 SSI nodes. The building is facing the railway track with its narrow side, which is $8 \mathrm{~m}$ wide, and the building is supported by two columns at either side. The length of the building is $10 \mathrm{~m}$ in the direction orthogonal to the track. In addition to the four columns placed at the corners, a column is placed in the middle of each of the longer sides. Each storey is $4 \mathrm{~m}$ high. The whole building structure is

Table 2. Railway track properties.

\begin{tabular}{|l|c|l|}
\hline Rail mass per unit length & 60.0 & $\mathrm{~kg} / \mathrm{m}$ \\
\hline Rail bending stiffness & $6.4 \cdot 10^{6}$ & $\mathrm{~N} / \mathrm{m}^{2}$ \\
\hline Rail loss factor & 0.01 & - \\
\hline Railpad stiffness & $5.0 \cdot 10^{8}$ & $\mathrm{~N} / \mathrm{m}$ \\
\hline Railpad loss factor & 0.1 & - \\
\hline Sleeper spacing & 1.0 & $\mathrm{~m}$ \\
\hline Sleeper mass per unit length & 542.0 & $\mathrm{~kg} / \mathrm{m}$ \\
\hline Ballast vertical stiffness & $4.64 \cdot 10^{9}$ & $\mathrm{~N} / \mathrm{m}^{2}$ \\
\hline Ballast mass per unit length & 1740 & $\mathrm{~kg} / \mathrm{m}$ \\
\hline Ballast loss factor & 0.04 & - \\
\hline Track width & 3.2 & $\mathrm{~m}$ \\
\hline
\end{tabular}


Figure 5. Investigated example case. The shades of colour indicate the vertical displacements (bright yellow is up, dark blue is down). The red nodes are the observation points, while the magenta nodes indicate the positions of the vehicle wheel sets placed on the exaggerated vertical rail profile.

constructed from concreate with a Young's modulus of $30 \mathrm{GPa}$, a Poisson's ratio of 0.15 , a mass density of $2400 \mathrm{~kg} / \mathrm{m}^{3}$, and loss factor of 0.03 . The columns have square cross-sections, with one side equal to $0.3 \mathrm{~m}$, while the floors are $0.25 \mathrm{~m}$ thick slabs. 3D beam elements were used to model the columns, using EulerBernoulli beam theory to account for bending. The floors of the building were modelled using MindlinReissner shell elements, accounting for bending as well as shear. All FE parts of the model were discretized with mesh size of $0.5 \mathrm{~m}$, resulting in 4841 degrees of freedom in the system. Three points on the building structure were created, where the displacements and velocities are observed, as shown in Figure 5. The excitation of the building structure is observed within the frequency range 1-60 Hz, with 400 discrete fixedFOR frequencies. The assembly of required matrices and solution of the full system takes around around 5 hours using a computer with double Intel Xeon E5-2620 CPUs.

Figure 5 shows the system response in time domain at the instance where the centre of the vehicle is at the position right in front of the centre of the building. The positions of the vehicle wheels are indicated by magenta coloured nodes placed on the uneven track surface. The black line shows the exaggerated track surface profile, and it also indicates location of the railway track centre. It can be observed that the displacements from the deadweight of the vehicle have the largest effect on the system, especially near the track structure. However, the building is further away from the track and is affected more by the lower-amplitude, higher-frequency excitation. This is especially evident when the velocities in the frequency domain of the first and second floors are investigated, as shown in Figure 6. Here, it can be observed that, while the low-frequency response is still
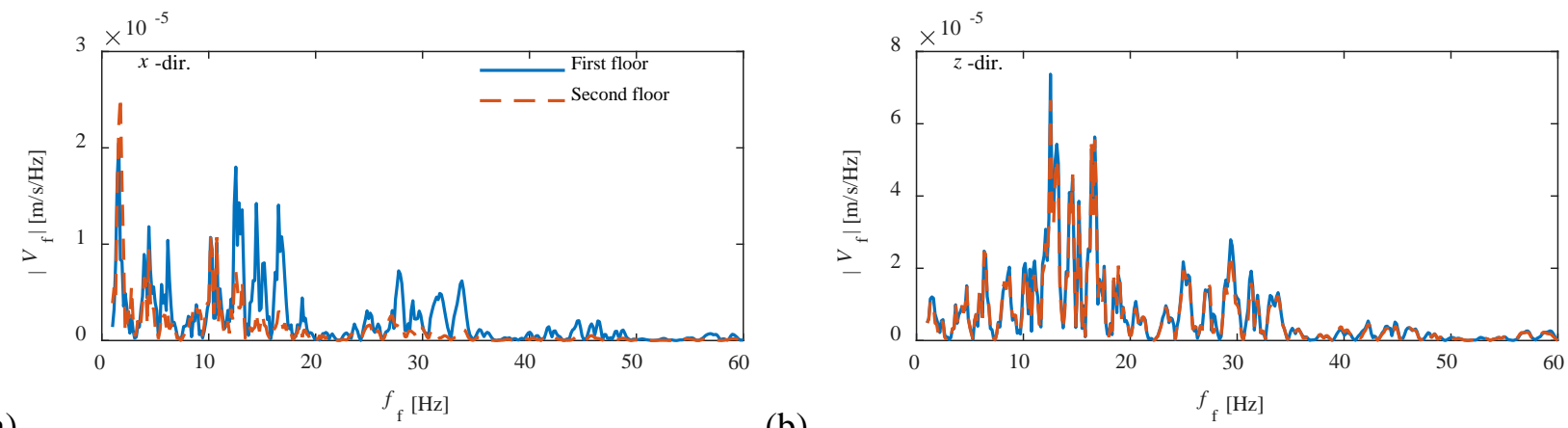

(a)

(b)

Figure 6. Velocities of the building structure in the frequency domain at the centre of the first and the second floors, (a) in $x$-direction; (b) in z-direction. 


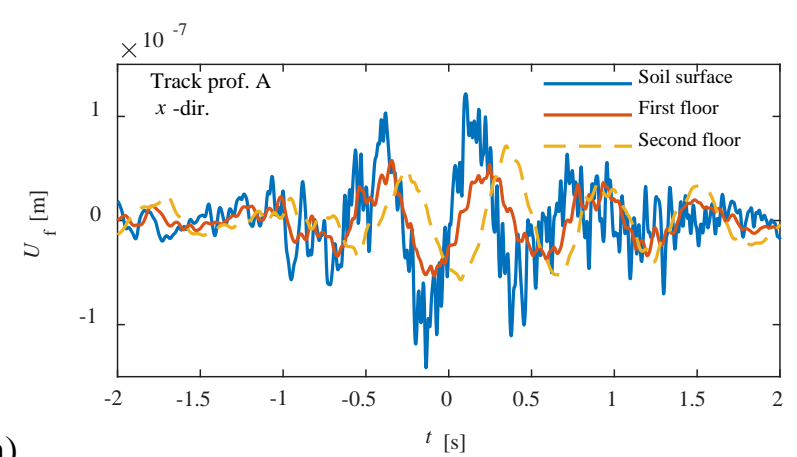

(a)

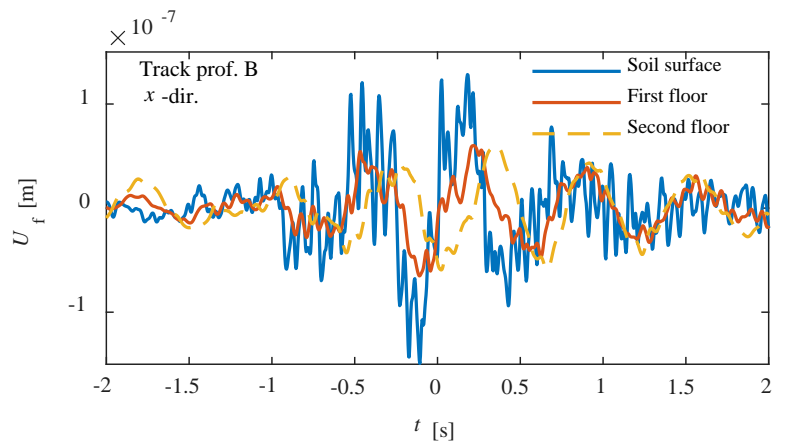

(c)

Figure 7. Displacements of the structure in the time domain generated from different rail unevenness profiles, (a) unevenness profile A and response in $x$-direction; (b) unevenness profile A, response in $z$ direction; (c) unevenness profile $\mathrm{B}$, response in $x$-direction; (d) unevenness profile $\mathrm{B}$, response in $z$-direction.

excited by the deadweight of the vehicle, higher-frequency excitation plays an important role-especially for the vertical velocities.

Comparing the response on the first and the second floors of the building, it can be observed that in the vertical direction both floors are excited almost identically to each other, while the differences for the horizontal $x$ direction (the vehicle travelling direction) are much larger. This is due to the relatively large axial stiffness of the columns, which propagates the excitation in the vertical direction well, exciting both floors almost equally. While, the bending stiffness of the columns is smaller and therefore the upper floor is isolated, especially at higher frequencies. This is seen on the left subfigure in Figure 6, where the high-frequency response is smaller for the second floor when compared to the first floor.

The time domain response of the system is obtained by assigning random phase angles to the unevenness of the rail corresponding to each considered wavenumber. This way, a random vertical rail profile is created adding generality to the predicted system response. However, the random rail profile introduces uncertainty to the system input that propagates to the system response. The effect such statistical track unevenness data to the response predictions has been studied in [42] for the ground surface vibration.

Figure 7 shows the time-domain response of the soil surface and the two floors from two different profiles of the uneven track. It can be observed that the track profiles have a significant effect on the observed system displacements in time domain, especially for the observed high-frequency components. However, the low frequencies are dominated by the deadweight of the vehicle and not affected much by different rail profiles. Further, the building structure acts as a filter of higher frequency vibration, significantly reducing the observed displacements when compared to the soil surface. This is especially evident for the horizontal displacements, where different rail profiles have a much smaller effect on the time domain displacements.

The displacements of the soil surface are also affected by the building structure. Figure 8 shows the velocities of the soil from a model with a building structure compared to a free-field solution. The building structure 


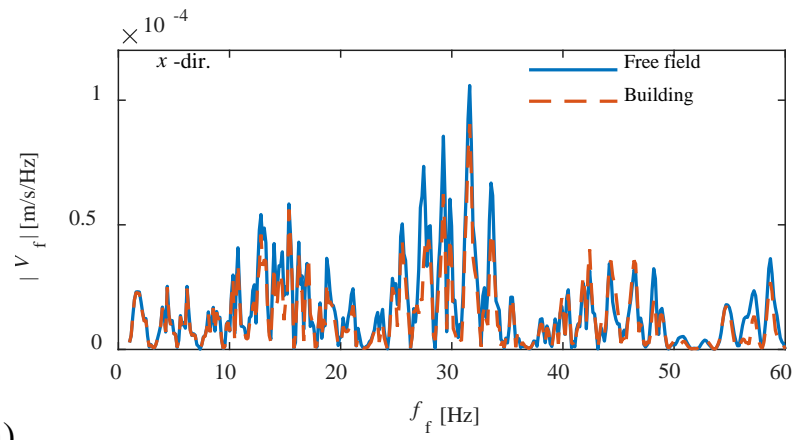

(a)

Figure 8. Velocities of the soil surface underneath the building compared to the free-field response, (a) response in $x$-direction; (b) response in $z$-direction.

reduces the observed velocities of the soil. However, the effects are not very large, with the largest change observed in the $10-35 \mathrm{~Hz}$ range. The frequency range corresponds well with the frequency range where the highest excitation of the building was obtained, as seen in Figure 6. Once again, these effects are more pronounced when analysing the horizontal displacements of the system.

\subsection{Fully coupled system}

A fully-coupled system solution approach might be necessary in cases where the modelled structures are close to the railway track, introducing a signifficant change of dynamic stiffness along the track. In that case, the rescattered waves can have an effect on the vehicle behaviour and the obtained wheel-rail interaction forces.

To examine such a case, a test case was set up modelling a single vehicle traveling across a railway track, as shown in Figure 9. The speed of the vehicle was $40 \mathrm{~m} / \mathrm{s}$. The vehicle and the track properties were the same as introduced in Tables 1 and 2. The soil was modelled as a half-space of clay, with material properties given in Section 6.1. Underneath the track, at a depth of $1 \mathrm{~m}$, a rigid block was embedded within the soil. The block was centred at the position, where the travelling vehicle centre line was located at time 0 . The block was modelled as a 2D plate, placed in the horizontal plane with one side equal to $2 \mathrm{~m}$. It was discretized into 36 discretization nodes, with three degrees of freedom per node. The system was excited by the deadweight of the vehicle only applied at $f_{\mathrm{m}}=0 \mathrm{~Hz}$, with no excitation from the rail unevenness. The system was assessed by the fully-coupled-modelling approach, described in Section 4.2. As only the quasi-static effects of the vehicle were modelled, the considered frequency ranges are reduced. The one-sided frequency range of the

Figure 9. Vehicle passing over a buried rigid block, at an instance where the leading wheel of the vehicle is directly above the centre of the rigid block. The vehicle is travelling at $40 \mathrm{~m} / \mathrm{s}$ from left to right. The black line indicates the vehicle traverse line, with the magenta nodes indicating the positions of the vehicle wheel sets. Only the two wheel sets of the front bogie are shown. 
moving FOR was $0-30 \mathrm{~Hz}$, and the one-sided frequency range of the fixed FOR is $0-25 \mathrm{~Hz}$. In the computation, negative as well as positive frequencies were considered for either FOR, and each range was split into 200 discrete frequencies. The obtained global receptance matrix size for the system, Eq. (27), is 22404 by 22404, which reduces to 4014 by 4014 global stiffness matrix, Eq. (35), when rigid structure condensation is performed. The solution time for the system is below 3 minutes, using a computer with double Intel Xeon E52620 CPUs.

Figure 10 shows the velocities obtained for the vehicle in the moving FOR as well as the rigid block in the fixed FOR. It can be observed that the vehicle passing over a rigid block introduces a significant excitation into the system, with the rigid block as well as the vehicle reacting to the passage. In this system, the observed excitation of the vehicle is purely due to the weak coupling effects that are disregarded in the two-step procedures. The waves generated by the passing vehicle are scattered by the rigid block in the fixed FOR and in turn excite a range of frequencies in the moving FOR. These effects would not be accounted for when the weak coupling between the vehicle and structures is not modelled. Thus, the partly coupled system assembly, as described in Section 4.1, would not produce any displacements to the parts of the vehicle, even though the rigid footing displacements would be practically the same Analysing the wheel velocities, as shown in Figure 10 top left, it can be seen that both leading wheels of separate bogies produce very similar results. However, the excitation due to the first wheel passage over the rigid block generates a wave that excites the third vehicle wheel, even before it reaches the rigid block. A similar effect is also observed after the third wheel passes over the rigid block, where the generated wave travels forward and excites the first vehicle wheel. A very similar behaviour is also observed on the bogies. Comparing these secondary excitations, it can be seen, that the reaction of the third wheel due to the first wheel passage is higher than the excitation of the first wheel due to third wheel passage. This is due to the Doppler effect of the wave travelling through the rail, as the third wheel is travelling towards the wave propagating form the first wheel, while the wave generated by the third
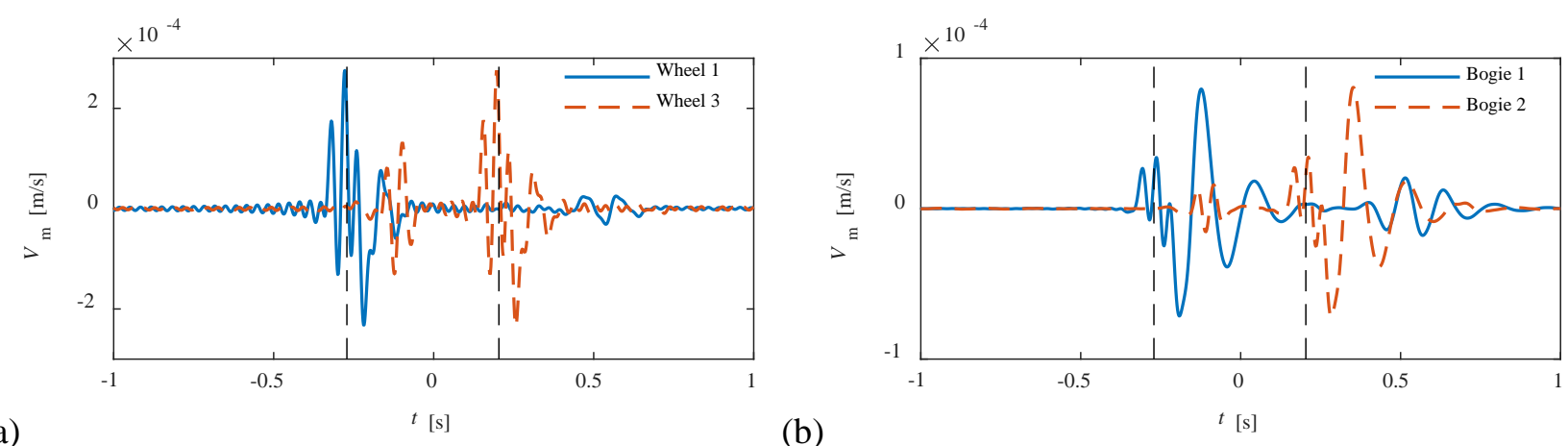

(a)

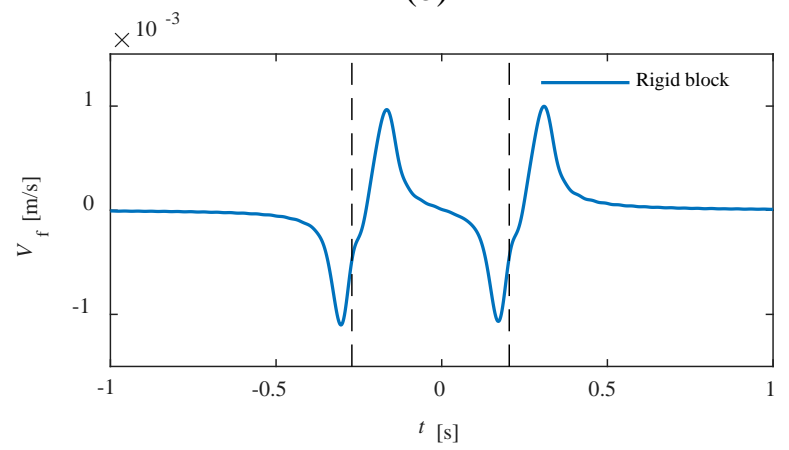

(c)

Figure 10. Vertical velocities for parts of the vehicle (in moving FOR) and the rigid block underneath the track (in fixed FOR). The vertical dashed black lines indicate time instance when the first and third vehicle wheels are directly above the centre of the rigid block. The response is shown for: (a) the first and the third vehicle wheels; (b) both vehicle bogies; (c) rigid block underneath the track. 
wheel must 'catch up' to the first wheel. Analysing the rigid block displacements, it can be observed that the largest velocities are reached just before the leading wheel of a bogie reaches the centre of block. Interestingly, to obtain the same displacements of the rigid block, a fully-coupled-system-solution approach is unnecessary, as the partly coupled solution provides results that are almost identical. For the analysed case, it can be concluded that the fully coupled solution approach is only necessary if the vehicle behaviour is of interest, for example for assessment of driver and passenger comfort, or if the track and wheel wear is to be assessed.

\section{Conclusions}

The paper introduced a new modelling approach for estimation of environmental vibration resulting from railway traffic. A model of a coupled vehicle-track-soil-building system was introduced with two approaches proposed for assembly and solution of the whole system: the partly-coupled approach and the fully-coupled approach. Both solution approaches use a single step procedure with one approach considering a fully-coupled system, while the other approach discards some secondary coupling effects for a more computationally efficient solution procedure. The presented modelling approach utilizes the commonly used moving-frame of reference formulation, however its capabilities are much larger. By utilizing both moving and stationary FORs, with analytically derived coupling terms, it allows to incorporate directly rigid and FE structures into the system. The obtained system is fully coupled, where it is possible to add additional structures and apply loads in either frame of reference simultaneously. Thus, complex phenomena, such as the effects of nearby structures to the wheel-rail interactions forces of a nearby passing vehicle, can be modelled, which is not possible with the commonly used moving-frame of reference approach.

A semi-analytical model was utilized to model the soil to which rigid objects and structures modelled by the FE were coupled. The proposed modelling approach uses the frequency-domain solution with some parts, such as the railway track, formulated in the moving FOR and other parts, such as building structures, formulated in a fixed FOR. The coupling terms between the two frames of reference are found by utilizing an analytical formulation of receptance between the two frames of reference. It has been established that due to the coupling between the fixed and moving frames of reference, the previously uncoupled discrete frequencies become coupled through the other FOR as a result of the Doppler effect and wave scattering. Modelling the system in two FORs simultaneously makes it possible to account for such effects, which would be otherwise disregarded in systems formulated in a single FOR. Additionally, using a railway track and a vehicle models formulated in a moving FOR allows the usage of computationally efficient analytical and semi-analytical models, which also do not introduce any reflected waves from the modelled domain boundaries.

The proposed methodology is a robust approach that does not suffer from numerical instabilities, due to the usage of frequency domain solutions. A wide range of cases can be assessed, including the modelling of rigid objects in or on the ground and flexible structures modelled by the FEM, interacting with the soil. Using the proposed partly-coupled-solution approach, the computations can be easily parallelized, thus providing a relatively fast and efficient computational method. Further, the suggested fully-coupled-solution procedure is useful in cases where the weak coupling between the vehicle and structures cannot be discarded.

In this work, a railway track with a train was considered as an example case. However, the methodology can be easily expanded to other cases, e.g. modelling a road with road traffic exciting the system. Further, adding FE models to the formulation is possible in the moving FOR as well as the fixed FOR. Thus, a more detailed railway track model or vehicle could also be added to the system.

The analytically derived coupling terms between the two frames of reference were validated by comparing with models formulated in a single FOR. Further, the partly coupled solution procedure of the full system was compared to a solution procedure in which the weak coupling is completely discarded. The two solution procedures have been found to provide almost identical results, validating the proposed partly coupled solution procedure while, at the same time, indicating insignificant back-coupling in the considered case. 
Finally, to demonstrate the capabilities of the numerical model, a building structure with rigid surface footings was analysed. The vibration levels at different floors were determined, as well as the effect of the structure on the surrounding soil, compared to a free-field solution. To demonstrate the potential of the fully-coupledsolution procedure, the response of a vehicle passing over a buried rigid block were investigated. It was been found that the fully coupled solution procedure can predict the weak coupling effects between the vehicle and the structure, producing a vehicle response distributed through frequencies, when the load is applied only at a single frequency. Thus, in the present example, the modes of the vehicle were excited parametrically by the waves scattered from the rigid inclusion as a result of the passing deadweight of the vehicle.

\section{Acknowledgements}

Part of the research was carried out in the framework of the project "Urban Tranquility" under the Interreg V programme. The first and the fourth authors of this work gratefully acknowledge the European Regional Development Fund for the financial support. Further, the first author would also like to acknowledge the generous support of the Graduate School of Science and Technology in Aarhus University. The second and third authors gratefully acknowledge the support of the EPSRC under the programme grant EP/M025276/1, 'The science and analytical tools to design long life, low noise railway track systems (Track to the Future)'.

\section{References}

[1] D.P. Connolly, G.P. Marecki, G. Kouroussis, I. Thalassinakis, P.K. Woodward, The growth of railway ground vibration problems — A review, Science of the Total Environment. 568 (2016) 1276-1282.

[2] High-Speed Ground Transportation Noise and Vibration Impact Assessment, Federal Railroad Administration, 2012.

[3] G. Degrande, G. Lombaert, An efficient formulation of Krylov's prediction model for train induced vibrations based on the dynamic reciprocity theorem, The Journal of the Acoustical Society of America. 110 (2002) 1379-1390.

[4] V. V. Krylov, F. Colin, Calculation of low-frequency ground vibrations from railway trains, Applied Acoustics. 42 (1994) 199-213.

[5] V. V. Krylov, Effects of Track Properties on Ground Vibrations Generated by High-Speed Trains, Acta Acustica. 228 (1998) 78-90.

[6] W.T. Thomson, Transmission of elastic waves through a stratified solid medium, Journal of Applied Physics. 21 (1950) 89-93.

[7] N.A. Haskell, The dispersion of surface waves on multilayered media, Bulletin of the Seismological Society of America. 43 (1953) 17-43.

[8] L.V. Andersen, J. Clausen, Impedance of surface footings on layered ground, Computers and Structures. 86 (2008) 72-87.

[9] P. Bucinskas, L.V. Andersen, Semi-analytical approach to modelling the dynamic behaviour of soil excited by embedded foundations, Procedia Engineering. 199 (2017) 2621-2626.

[10] X. Sheng, C.J.C. Jones, M. Petyt, Ground vibration generated by a harmonic load acting on a railway track, Journal of Sound and Vibration. 225 (1999) 3-28.

[11] X. Sheng, C.J.C. Jones, M. Petyt, Ground vibration generated by a load moving along a railway track, Journal of Sound and Vibration. 228 (1999) 129-156.

[12] X. Sheng, C.J.C. Jones, D.J. Thompson, A theoretical model for ground vibration from trains generated by vertical track irregularities, Journal of Sound and Vibration. 272 (2004) 937-965. 
[13] W. Zhai, Z. He, X. Song, Prediction of high-speed train induced ground vibration based on train-trackground system model, Earthquake Engineering and Engineering Vibration. 9 (2010) 545-554.

[14] D.P. Connolly, A. Giannopoulos, M.C. Forde, Numerical modelling of ground borne vibrations from high speed rail lines on embankments, Soil Dynamics and Earthquake Engineering. 46 (2013) 13-19.

[15] G. Kouroussis, O. Verlinden, Prediction of railway induced ground vibration through multibody and finite element modelling, Mechanical Sciences. 4 (2013) 167-183.

[16] J. O’Brien, D.C. Rizos, A 3D BEM-FEM methodology for simulation of high speed train induced vibrations, Soil Dynamics and Earthquake Engineering. 25 (2005) 289-301.

[17] L.V. Andersen, C.J.C. Jones, Coupled boundary and finite element analysis of vibration from railway tunnels-a comparison of two- and three-dimensional models, Journal of Sound and Vibration. 293 (2006) 611-625.

[18] P. Galvín, A. Romero, J. Domínguez, Fully three-dimensional analysis of high-speed train-track-soilstructure dynamic interaction, Journal of Sound and Vibration. 329 (2010) 5147-5163.

[19] D. Aubry, D. Clouteau, G. Bonnet, Modelling of wave propagation due to fixed or mobile dynamic sources, Workshop Wave. 94 (1994) 109-121.

[20] Y. Bin Yang, H.H. Hung, A 2.5D finite/infinite element approach for modelling visco-elastic bodies subjected to moving loads, International Journal for Numerical Methods in Engineering. 51 (2001) $1317-1336$.

[21] X. Sheng, C.J.C. Jones, D.J. Thompson, Prediction of ground vibration from trains using the wavenumber finite and boundary element methods, Journal of Sound and Vibration. 293 (2006) 575586.

[22] Q. Jin, D.J. Thompson, D.E.J. Lurcock, M.G.R. Toward, E. Ntotsios, A 2.5D finite element and boundary element model for the ground vibration from trains in tunnels and validation using measurement data, Journal of Sound and Vibration. 422 (2018) 373-389.

[23] P. Lopes, P.A. Costa, M. Ferraz, R. Calçada, A.S. Cardoso, Numerical modeling of vibrations induced by railway traffic in tunnels: From the source to the nearby buildings, Soil Dynamics and Earthquake Engineering. 61-62 (2014) 269-285.

[24] A. Yaseri, M.H. Bazyar, S. Javady, 2.5D coupled FEM-SBFEM analysis of ground vibrations induced by train movement, Soil Dynamics and Earthquake Engineering. 104 (2018) 307-318.

[25] G. Kouroussis, D.P. Connolly, O. Verlinden, Railway-induced ground vibrations - a review of vehicle effects, International Journal of Rail Transportation. 2 (2014) 69-110.

[26] D.J. Thompson, G. Kouroussis, E. Ntotsios, Modelling, simulation and evaluation of ground vibration caused by rail vehicles, Vehicle System Dynamics. 57 (2019) 936-983.

[27] D.P. Connolly, G. Kouroussis, O. Laghrouche, C.L. Ho, M.C. Forde, Benchmarking railway vibrations - Track, vehicle, ground and building effects, Construction and Building Materials. 92 (2015) 64-81.

[28] P. Fiala, G. Degrande, F. Augusztinovicz, Numerical modelling of ground-borne noise and vibration in buildings due to surface rail traffic, Journal of Sound and Vibration. 301 (2007) 718-738.

[29] S. François, L. Pyl, H.R. Masoumi, G. Degrande, The influence of dynamic soil-structure interaction on traffic induced vibrations in buildings, Soil Dynamics and Earthquake Engineering. 27 (2007) 655674.

[30] G. Kouroussis, L. Van Parys, C. Conti, O. Verlinden, Prediction of ground vibrations induced by urban railway traffic: an analysis of the coupling assumptions between vehicle, track, soil, and buildings, 
International Journal of Acoustics and Vibration. 163 (2013) 163-172.

[31] M.F.M. Hussein, H. Hunt, K. Kuo, P.A. Costa, J. Barbosa, The use of sub-modelling technique to calculate vibration in buildings from underground railways, Proceedings of the Institution of Mechanical Engineers, Part F: Journal of Rail and Rapid Transit. 229 (2015) 303-314.

[32] D. López-Mendoza, A. Romero, D.P. Connolly, P. Galvín, Scoping assessment of building vibration induced by railway traffic, Soil Dynamics and Earthquake Engineering. 93 (2017) 147-161.

[33] D.P. Connolly, P. Galvín, B. Olivier, A. Romero, G. Kouroussis, A 2.5D time-frequency domain model for railway induced soil-building vibration due to railway defects, Soil Dynamics and Earthquake Engineering. 120 (2019) 332-344.

[34] M.F.M. Hussein, H.E.M. Hunt, A numerical model for calculating vibration from a railway tunnel embedded in a full-space, Journal of Sound and Vibration. 305 (2007) 401-431.

[35] J.Freisinger, M. Hackenberg, G. Müller, A coupled Integral Transform Method - Finite Element Method approach to model the Soil Structure Interaction of finite (3D) and length invariant (2.5D) systems, Journal of Sound and Vibration. 482 (2020) 115443.

[36] E. Kausel, J.M. Roesset, Stiffness matrices for layered soils, Bulletin of the Seismological Society of America. 71 (1981) 1743-1761.

[37] R. Wang, A simple orthonormalization method for stable and efficient computation of Green's functions, Bulletin of the Seismological Society of America. 89 (1999) 733-741.

[38] Z. Han, M. Zhou, X. Zhou, L. Yang, Dynamic Response of 3D Surface/Embedded Rigid Foundations of Arbitrary Shapes on Multi-Layered Soils in Time Domain, International Journal of Structural Stability and Dynamics. 19 (2019).

[39] E. Ntotsios, D.J. Thompson, M.F.M. Hussein, The effect of track load correlation on ground-borne vibration from railways, Journal of Sound and Vibration. 402 (2017) 142-163.

[40] P. Coulier, G. Lombaert, G. Degrande, The influence of source-receiver interaction on the numerical prediction of railway induced vibrations, Journal of Sound and Vibration. 333 (2014) 2520-2538.

[41] D. Cantero, T. Arvidsson, E. OBrien, R. Karoumi, Train-track-bridge modelling and review of parameters, Structure and Infrastructure Engineering. 12 (2016) 1051-1064.

[42] G. Lombaert, P. Galvín, S. François, G. Degrande, Quantification of uncertainty in the prediction of railway induced ground vibration due to the use of statistical track unevenness data, Journal of Sound and Vibration. 333 (2014) 4232-4253. 